\title{
Star-Triangle Relations in the Exactly Solvable Statistical Models
}

\author{
S. V. Pokrovsky and Yu. A. Bashilov \\ Space Research Institute, Academy of Sciences, SU-117810, Moscow, USSR
}

\begin{abstract}
A regular method for analysis of lattice spin models with a nearest neighbour interaction is proposed. Star-triangle relations in the form of functional equations are used. Parametric families of transfer matrices commuting due to star-triangle relations are constructed. The eigenvalues of transfer matrices as functions of the spectral parameter are shown to obey two functional equations. The solution of these equations for the maximal eigenvalue yields the partition function of the model. The method is applied for evaluation of the partition function of the critical Potts models, the Ising model, the Ashkin-Teller model equivalent to the eight-vertex model.
\end{abstract}

\section{Introduction}

The star-triangle transform has served for the analysis of the exactly solvable lattice statistical spin systems for a long time. It was briefly mentioned in the famous work by Onsager [1] and used in his article [2]. Star-triangle relations (STR) combined with dual transformation (DT) made it possible to determine the critical point of the Ising models on the triangular and honeycomb lattices [3-6]. Baxter and Enting evaluated the partition function of the Ising model using only STR [7]. STR were also utilized to construct the exact renormalization group equations for the Ising model on the triangular lattice $[8,9]$. Mittag and Stephen [10] applied STR to the critical Potts models [11].

In the present paper we consider STR as functional equations. Their derivation is based on the Yang-Baxter equations of triangles (ET) [12-15]. The solutions of STR are used to construct parametric families of commuting transfer matrices (TM). These TM are ascertained to be normal operators. This allows us in principle to diagonalize the whole family by a single unitary transformation.

To find the eigenvalues of TM concerned one might use them to construct a Baxter-type TM [13] and then employ the generalized Bethe-ansatz technique. The latter was proposed by Baxter and elaborated to a standard procedure in $[16,17]$. Unfortunately, the search of pseudo-vacuum that is the first step of this procedure 
has not been canonized yet. Only some particular solutions of the problem are known so far [18].

For this reason we use an alternative approach in the present paper. It is based on two functional equations that rule the behaviour of TM eigenvalues as functions of the spectral parameter. The free energy of the model is determined by these equations uniquely assuming its analyticity and periodicity.

The above method is applied to evaluate the partition function of all the spin models on the rectangular lattice that are known to be exactly solvable. The analogous approach for the vertex statistical Baxter-type models is developed in $[15,19]$.

This paper is organized as follows. General theorems and equations are considered in Sects. 2-6 while Sects. 7-9 are devoted to particular models. Section 2 serves to introduce basic ET in two interpretations : $1+1$-dimensional scattering theory and the spin statistical model. This comparison makes it natural to consider ET as functional equations. In the same section STR are derived from ET. The corollaries of STR called unitarity conditions are examined. In Sect. 3 we use the solutions of STR to construct TM depending on the spectral parameter. A theorem of commutativity for TM with different values of the spectral parameter is proved. In Sect. 4 the diagonalizability of TM is discussed. The functional equations for its eigenvalues are found with the help of unitarity conditions. Section 5 is devoted to the symmetry of STR and ET under dual transformations. In Sect. 6 some of the above results are formulated in the operator language. In the thermodynamic limit $\mathrm{TM}$ is a generating functional of an infinite series of quantum local conservation laws [20]. The solutions of STR are found: in Sect. 7 for the critical Potts models, in Sect. 8 for the Ising model, and in Sect. 9 for the restricted Ashkin-Teller model. The evaluation of the partition functions of the models involved is represented in Sect. 10. The most cumbersome calculations of Sects. 7, 8 are given in Appendices A, B. Some formulas concerning the eight-vertex model are collected in Appendix C.

\section{Equations of Triangles and Star-Triangle Relations}

Consider a relativistic scattering theory including $n$ kinds of particles with equal masses in $1+1$ space-time dimensions. The collision of two particles with rapidities $\theta_{1}$ and $\theta_{2}$ is described by a scattering matrix $S_{\alpha_{1} \alpha_{2}}^{\beta_{1} \beta_{2}}\left(\theta_{12}\right)$ (Fig. 1). The indices $\alpha_{1,2}$

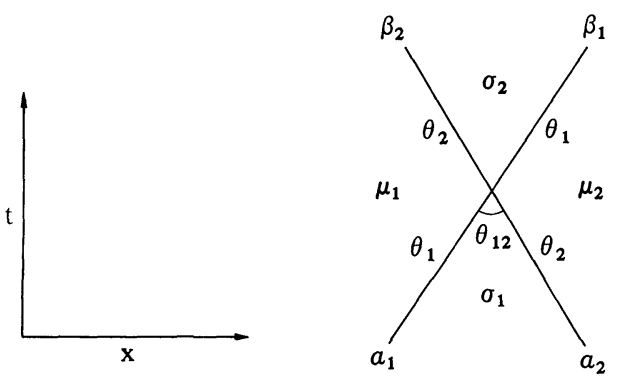

Fig. 1. A diagram of two-particle scattering. Imaginary time is directed along the t-axis. Imaginary relative rapidity is denoted by $\theta_{12}$ 


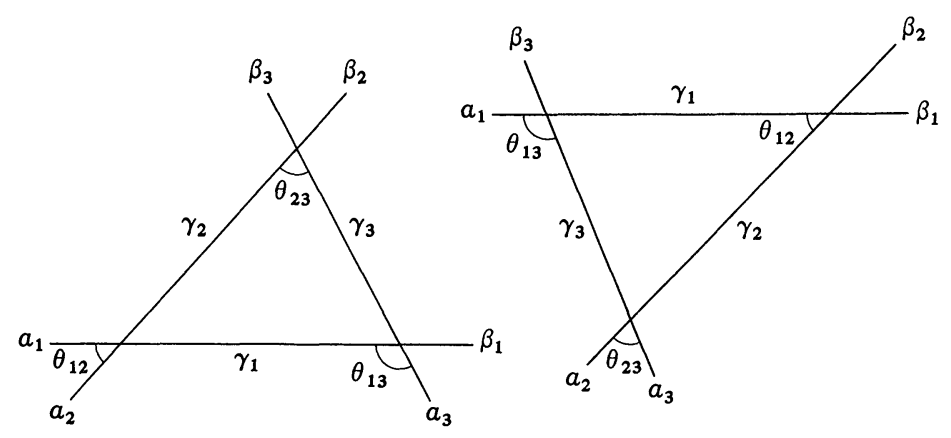

Fig. 2. Two possible variants of three-particle scattering. A graphical representation of equations of triangles $(2.1)$

denote the kinds of colliding particles while $\beta_{1,2}$ specify emitted particles. A pair of rapidities $\theta_{1}$ and $\theta_{2}$ remains unchanged in two-particle scattering due to the identity of masses of the particles and to energy and momentum conservation. $S$-matrix depends only on the difference $\theta_{12}=\theta_{1}-\theta_{2}$ on account of Lorentz-invariance.

If a system possesses an infinite series of conservation laws, a set of individual rapidities is conserved in any multiparticle collision. A number of works [15,21-23] deals with $S$-matrices that may be represented as a product of two-particle amplitudes. The resulting amplitude of the $S$-matrix must be independent of the sequence of two-particle collisions. This is valid for any multiparticle amplitude if and only if three-particle $S$-matrix elements constructed from two-particle amplitudes in different ways are equal $[12,15]$. Two possible variants are presented in Fig. 2. The equalities of the depicted amplitudes are the factorization equations also called equations of triangles (ET) [23]:

$$
\begin{gathered}
S_{\alpha_{1} \alpha_{2}}^{\gamma_{1} \gamma_{2}}\left(\theta_{12}\right) S_{\gamma_{1} \alpha_{3}}^{\beta_{1} \gamma_{3}}\left(\theta_{13}\right) S_{\gamma_{2} \gamma_{3}}^{\beta_{2} \beta_{3}}\left(\theta_{23}\right) \\
=S_{\alpha_{2} \alpha_{3}}^{\gamma_{2} \gamma_{3}}\left(\theta_{23}\right) S_{\alpha_{1} \gamma_{3}}^{\gamma_{1} \beta_{3}}\left(\theta_{13}\right) S_{\gamma_{1} \gamma_{2}}^{\beta_{1} \beta_{2}}\left(\theta_{12}\right), \\
\theta_{i k}=\theta_{i}-\theta_{k}, \quad 0 \leqq \alpha_{i}, \beta_{i}, \gamma_{i}<n, \quad i=1,2,3 .
\end{gathered}
$$

The summation over repeating indices is implied.

The absence of scattering of particles with equal rapidities results in the following natural initial conditions for the $S$-matrix [21]:

$$
S_{\alpha_{1} \alpha_{2}}^{\beta_{1} \beta_{2}}(0)=\delta_{\alpha_{1}, \beta_{2}} \delta_{\alpha_{2}, \beta_{1}} .
$$

The conditions (2.2), further referred to as standard initial conditions, are compatible with ET (2.1) provided that

$$
S_{\alpha_{1} \alpha_{2}}^{\gamma_{1} \gamma_{2}}(\theta) S_{\gamma_{2} \gamma_{1}}^{\beta_{2} \beta_{1}}(-\theta)=\delta_{\alpha_{1}, \beta_{1}} \delta_{\alpha_{2}, \beta_{2}} F(\theta)
$$

Equation (2.3) are known as unitarity conditions (UC) [15].

ET (2.1) admit the following symmetry restriction imposed on the $S$-matrix : the crossing symmetry condition

$$
S_{\alpha_{1} \alpha_{2}}^{\beta_{1} \beta_{2}}(\theta)=S_{\alpha_{2} \beta_{1}^{*}}^{\beta_{2} \alpha_{1}^{*}}(i \pi-\theta),
$$


PT-invariance

$$
S_{\alpha_{1} \alpha_{2}}^{\beta_{1} \beta_{2}}(\theta)=S_{\beta_{2} \beta_{1}}^{\alpha_{2} \alpha_{1}}(\theta)
$$

CP-invariance

$$
S_{\alpha_{1} \alpha_{2}}^{\beta_{1} \beta_{2}}(\theta)=S_{\alpha_{2} \alpha_{1}^{*}}^{\beta_{2}^{*} \beta_{1}^{*}}(\theta) \text {. }
$$

The asterisk in formulas (2.4) and (2.6) denotes charge conjugation.

The sort of particle is adopted to be specified by its isotopic charge that is conserved modulo $n$. In two-particle scattering:

$$
\alpha_{1}+\alpha_{2}=\beta_{1}+\beta_{2}(\bmod n)
$$

Define charge conjugation as a reversal of its sign:

$$
\alpha^{*}=-\alpha(\bmod n) \text {. }
$$

$S$-matrices obeying the above conditions are well suited to describe twodimensional spin statistical systems with four-spin interaction. Assume the rapidities to be pure imaginary. Then their differences acquire geometrical meaning of angles depicted in Figs. 1 and 2. If the vertices of the same type as in Fig. 1 form a lattice, the plaquettes of the latter may be occupied by spin variables $\sigma$ and $\mu$ (Fig. 1) in a way to satisfy condition (2.7):

$$
\alpha_{1}=\sigma_{1}-\mu_{1} ; \quad \alpha_{2}=\mu_{2}-\sigma_{1} ; \quad \beta_{1}=\mu_{2}-\sigma_{2} ; \quad \beta_{2}=\sigma_{2}-\mu_{1} .
$$

The addition of indices in the present and further formulas is implied modulo $n$.

The $S$-matrix element may be regarded as a statistical weight (SW) of a corresponding spin configuration:

$$
S_{\alpha_{1} \alpha_{2}}^{\beta_{1} \beta_{2}}(\theta)=R_{\mu_{1} \mu_{2}}^{\sigma_{1} \sigma_{2}}(-i \theta) .
$$

Equations (2.9) and (2.10) show that $R_{\mu_{1} \mu_{2}}^{\sigma_{1} \sigma_{2}}(\theta)$ depends only on the relative orientation of spins.

The requirements (2.4)-(2.6) appear to be rather natural in terms of the spin model. Namely, PT-invariance (2.5) is equivalent to the symmetry in the indices $\sigma_{1}$ and $\sigma_{2}$ :

$$
R_{\mu_{1} \mu_{2}}^{\sigma_{1} \sigma_{2}}(\theta)=R_{\mu_{1} \mu_{2}}^{\sigma_{2} \sigma_{1}}(\theta)
$$

while CP-invariance (2.6) converts into the symmetry in the indices $\mu_{1}$ and $\mu_{2}$ :

$$
R_{\mu_{1} \mu_{2}}^{\sigma_{1} \sigma_{2}}(\theta)=R_{\mu_{2} \mu_{1}}^{\sigma_{1} \sigma_{2}}(\theta) .
$$

The condition of crossing (2.4) takes the following form:

$$
R_{\mu_{1} \mu_{2}}^{\sigma_{1} \sigma_{2}}(\theta)=R_{\sigma_{1} \sigma_{2}}^{\mu_{1} \mu_{2}}(\pi-\theta) .
$$

ET (2.1) in terms of spin variables, taking (2.9)-(2.13) into account, are written as follows :

$$
\begin{aligned}
\sum_{\sigma=0}^{n-1} R_{\mu_{2} \mu_{3}}^{\sigma_{1} \sigma}\left(\theta_{1}\right) R_{\mu_{3} \mu_{1}}^{\sigma_{2} \sigma}\left(\theta_{2}\right) R_{\mu_{1} \mu_{2}}^{\sigma_{3} \sigma}\left(\theta_{3}\right) & =\sum_{\mu=0}^{n-1} R_{\sigma_{2} \sigma_{3}}^{\mu_{1} \mu}\left(\theta_{1}\right) R_{\sigma_{3} \sigma_{1}}^{\mu_{2} \mu}\left(\theta_{2}\right) R_{\sigma_{1} \sigma_{2}}^{\mu_{3} \mu}\left(\theta_{3}\right), \\
\theta_{1}+\theta_{2}+\theta_{3} & =\pi .
\end{aligned}
$$

Equation (2.14) are graphically represented in Fig. 3. 


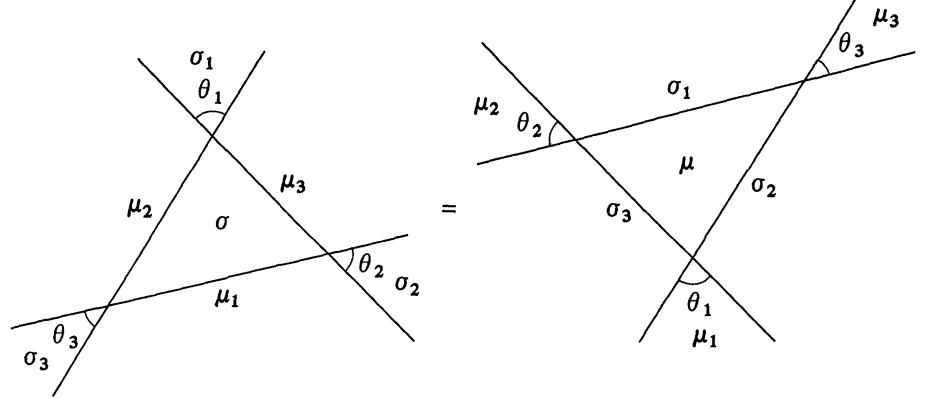

Fig. 3. Equations of triangles in terms of spin (plaquette) variables

As Baxter has shown $[13,14]$, if $(2.14)$ is satisfied, the partition function of the spin model is invariant under parallel translations of the direct lines forming the lattice, thus depending only on the angles between them. This property is known as $Z$-invariance [14].

From standard initial conditions (2.2) with the help of (2.9) and (2.13) one can deduce standard boundary conditions (SBC):

$$
R_{\mu_{1} \mu_{2}}^{\sigma_{1} \sigma_{2}}(0)=\delta_{\sigma_{1}, \sigma_{2}}, \quad R_{\mu_{1} \mu_{2}}^{\sigma_{1} \sigma_{2}}(\pi)=\delta_{\mu_{1}, \mu_{2}} .
$$

The analogue of $\mathrm{UC}(2.3)$ is readily obtained from (2.14) if one of the arguments $\theta_{i}$ is put equal to $\pi$ and (2.15) is used:

$$
\sum_{\sigma=0}^{n-1} R_{\mu_{1} \mu_{2}}^{\sigma_{1} \sigma}(\theta) R_{\mu_{1} \mu_{2}}^{\sigma_{2} \sigma}(-\theta)=\delta_{\sigma_{1}, \sigma_{2}} F(\theta) .
$$

Equation (2.16) must hold for arbitrary $\mu_{1}$ and $\mu_{2} . F(\theta)$ is an even function of $\theta$.

The spin model under consideration is actually built of two types of spins $\sigma$ and $\mu$, each type situated on its own sublattice [14] (Fig. 3). The absence of interaction between different sublattice spins corresponds to the following factorization of SW $R_{\mu_{1} \mu_{2}}^{\sigma_{1} \sigma_{2}}$ into the product:

$$
R_{\mu_{1} \mu_{2}}^{\sigma_{1} \sigma_{2}}(\theta)=K_{\sigma_{1} \sigma_{2}}(\theta) K_{\mu_{1} \mu_{2}}(\pi-\theta)
$$

The factorized $R$-matrix (2.17) obviously obeys the crossing condition (2.13) while restrictions (2.11) and (2.12) mean that SW $K_{\sigma_{1} \sigma_{2}}(\theta)$ is symmetric in its indices.

Substituting (2.17) into (2.14) one comes to star-triangle relation (STR):

$$
\begin{aligned}
& \sum_{\sigma=0}^{n-1} K_{\sigma_{1} \sigma}\left(\theta_{1}\right) K_{\sigma_{2} \sigma}\left(\theta_{2}\right) K_{\sigma_{3} \sigma}\left(\theta_{3}\right) \\
& \quad=\lambda\left(\theta_{1}, \theta_{2}, \theta_{3}\right) K_{\sigma_{2} \sigma_{3}}\left(\pi-\theta_{1}\right) K_{\sigma_{3} \sigma_{1}}\left(\pi-\theta_{2}\right) K_{\sigma_{1} \sigma_{2}}\left(\pi-\theta_{3}\right),
\end{aligned}
$$

where

$$
\theta_{1}+\theta_{2}+\theta_{3}=\pi
$$

and $\lambda\left(\theta_{1}, \theta_{2}, \theta_{3}\right)$ is some function symmetric in its arguments.

We introduce a graphical representation of (2.18) denoting $K_{\sigma_{1} \sigma_{2}}(\theta)$ by the line connecting the points $\sigma_{1}$ and $\sigma_{2}$. Then Fig. 4 is equivalent to Eq. (2.18). 


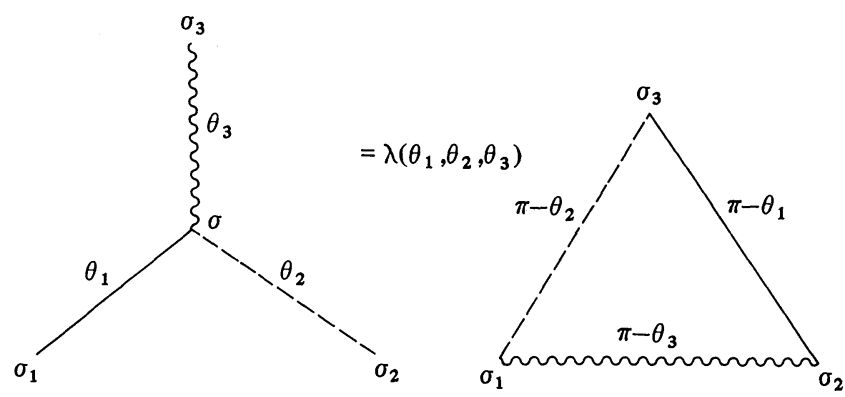

Fig. 4. Graphical representation of star-triangle relations (2.18). Summation over index $\sigma$ on the lefthand side is implied

SBC (2.15) due to (2.17) lead to the SBC for $K_{\sigma_{1} \sigma_{2}}(\theta)$ :

$$
K_{\sigma_{1} \sigma_{2}}(0)=v \delta_{\sigma_{1}, \sigma_{2}}, \quad K_{\sigma_{1} \sigma_{2}}(\pi)=v^{-1} .
$$

Setting one of the arguments $\theta_{i}$ in (2.18) equal to $\pi$ and taking into consideration (2.20) one arrives at a unitarity condition (UC) that is expressed now by two equations :

$$
\begin{gathered}
\sum_{\sigma} K_{\sigma_{1} \sigma}(\theta) K_{\sigma_{2} \sigma}(-\theta)=f(\theta) \quad \delta_{\sigma_{1}, \sigma_{2}}, \\
K_{\sigma_{1} \sigma_{2}}(\pi-\theta) K_{\sigma_{1} \sigma_{2}}(\pi+\theta)=g(\theta),
\end{gathered}
$$

where $f(\theta)$ and $g(\theta)$ are some even functions.

Note that any two solutions of STR (2.18) and UC (2.21), (2.22) differing by an arbitrary factor $\varrho(\theta)$ may be regarded as equivalent. Really, multiplication of $K_{\sigma_{1} \sigma_{2}}(\theta)$ by $\varrho(\theta)$ leads only to the renormalization of $\lambda\left(\theta_{1}, \theta_{2}, \theta_{3}\right)$ in $(2.18)$ and $f(\theta)$, $g(\theta)$ in $(2.21),(2.22)$, respectively.

\section{Construction and Commutativity of Transfer Matrices}

It was shown by Baxter [13] with the aid of ET (2.1), (2.14) that transfer matrices (TM) of $Z$-invariant models form parametric commutative families. The analogous feature is exhibited by spin models possessing STR. Namely, TM along the diagonal commute under certain boundary conditions. TM of this type were first introduced by Onsager [2] and later used by Mittag and Stephen [24].

We construct the diagonal TM $\mathbf{T}_{\{\mu, \sigma\}}$ using a graphical representation as a saw depicted in Fig. 5. The algebraic expression for its matrix elements is the following:

$$
\mathbf{T}_{\{\mu, \sigma\}}(\theta)=\prod_{i=1}^{N} K_{\sigma_{1} \mu_{i}}(\theta) K_{\sigma_{i+1} \mu_{i}}(\pi-\theta),
$$

where $\{\sigma\}=\left(\sigma_{1}, \sigma_{2}, \ldots, \sigma_{N}\right)$ and $\{\mu\}=\left(\mu_{1}, \mu_{2}, \ldots, \mu_{N}\right)$. Notice that a cyclic boundary condition is assumed:

$$
\sigma_{N+1}=\sigma_{1} .
$$

The parameter $\theta$ determines an anisotropy of the rectangular lattice model as it is seen in Fig. 5. An isotropy is achieved when $\theta=\pi / 2$. 

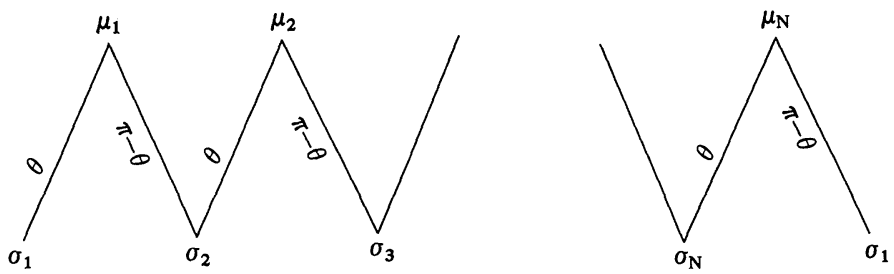

Fig. 5. Transfer-matrix (3.1)

The partition function of the spin system on the rectangular lattice containing $2 M$ saws as depicted in Fig. 5 is expressed via $\mathbf{T}_{\{\mu, \sigma\}}(\theta)$ and the transposed matrix $\mathbf{T}_{\{\mu, \sigma\}}^{\sim}(\theta)=\mathbf{T}_{\{\sigma, \mu\}}(\theta)$ in the following way:

$$
\mathbb{Z}=\operatorname{tr}\left[\mathbf{T}^{\sim}(\pi-\theta) \mathbf{T}(\theta)\right]^{M} .
$$

Using the construction of Fig. 5 with the cyclic boundary condition (3.2) and the symmetry of $K_{\sigma_{1} \sigma_{2}}(\theta)$ in its indices one can deduce

where

$$
\mathbf{T}(\pi-\theta)=\mathbf{P T}^{\sim}(\theta),
$$

$$
\mathbf{P}_{\{\mu, \sigma\}}=\prod_{i=1}^{N} \delta_{\sigma_{i+1}, \mu_{i}}, \quad \sigma_{N+1}=\sigma_{1}
$$

is an operator of the lattice spacing translation commuting with $\mathbf{T}(\theta)$ :

$$
[\mathbf{P}, \mathbf{T}(\theta)]=0 .
$$

We are in a position to show that for arbitrary $\theta$ and $\theta^{\prime}$

$$
\left[\mathbf{T}(\theta), \mathbf{T}\left(\theta^{\prime}\right)\right]=0 \text {. }
$$

Instead of Eq. (3.7) we shall prove another identity equivalent to (3.7) due to Eq. (3.6):

$$
\mathbf{T}^{\sim}\left(\pi-\theta^{\prime}\right) \mathbf{T}(\theta)=\mathbf{T}(\theta) \mathbf{T}^{\sim}\left(\pi-\theta^{\prime}\right)
$$

Equation (3.8) is represented graphically in Fig. 6 where the summation is implied over all indices $\lambda$ and $v$.

Multiply the product $\mathbf{T}^{\sim}\left(\pi-\theta^{\prime}\right) \mathbf{T}(\theta)$ in Fig. 6a by SW $K_{\sigma_{1} \mu_{1}}\left(\pi-\theta+\theta^{\prime}\right)$ designated by a wavy line. Consider the graphical equality in Fig. 7 which is simply STR in Fig. 4 repeated twice. Applying the equality of Fig. 7 successively to Fig. 6 one may carry the wavy line through the whole product $\mathbf{T}^{\sim}\left(\pi-\theta^{\prime}\right) \mathbf{T}(\theta)$ in Fig. 6a and finally obtain $\mathbf{T}(\theta) \mathbf{T}^{\sim}\left(\pi-\theta^{\prime}\right)$ in Fig. 6 b multiplied by SW $K_{\sigma_{N+1} \mu_{N+1}}\left(\pi-\theta+\theta^{\prime}\right)$. Due to the cyclic boundary conditions (3.2) the latter is equal to SW $K_{\sigma_{1} \mu_{1}}\left(\pi-\theta+\theta^{\prime}\right)$. Canceling the identical factors on both sides of Eq. (3.8) one completes the desirable proof.

The above proof may be easily converted into a purely algebraic one but the unwieldiness of the latter prevents us from producing it here. 

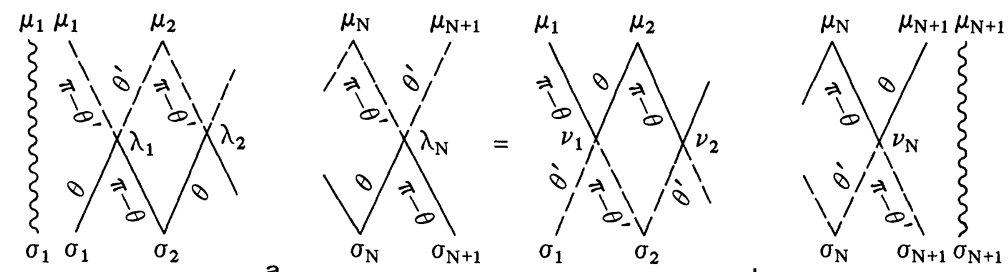

Fig. 6. a Product $\mathbf{T}^{\sim}\left(\pi-\theta^{\prime}\right) \mathbf{T}(\theta)$. b Product $\mathbf{T}(\theta) \mathbf{T}^{\sim}\left(\pi-\theta^{\prime}\right)$. TM $\mathbf{T}(\theta)$ is denoted by a solid line, $\mathbf{T}^{\sim}\left(\pi-\theta^{\prime}\right)$ by a dashed line. Summation over all indices $\lambda, v$ is implied

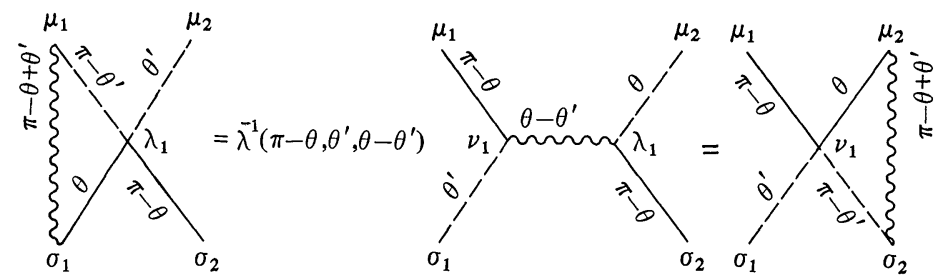

Fig. 7. Graphical identity used for the proof of commutativity of TM. Here STR (2.18) is repeated twice

\section{Functional Equations for TM Eigenvalues}

TM (3.1) is a positive linear operator acting in the $n^{N}$-dimensional Euclidean space. Really, the components of the vectors in this space are labeled by spin configurations in the row of the lattice and are equal to the corresponding SW. Therefore, they are real and positive. TM elements have the meaning of SW ascribed to the spin configurations in the two adjacent rows and so are real and positive as well. Besides, due to Eq. (3.8) $\mathbf{T}(\theta)$ is normal and according to the general theorems of linear algebra may be diagonalized simultaneously with its adjoint (transposed) operator $\mathbf{T}^{\dagger}(\theta)=\mathbf{T}^{\sim}(\theta)$. The eigenvalues of $\mathbf{T}(\theta)$ and $\mathbf{T}^{\sim}(\theta)$ corresponding to the same common eigenvector are complex conjugate [25, Chap. IX, Sect. 13].

Moreover, $\operatorname{TMT}(\theta)$ with permissible values of $\theta$ form the commutative family of normal operators. Therefore, there is some unitary transformation diagonalizing the whole family and consequently independent on $\theta$ [25, Chap. IX, Sect. 15].

SBC (2.20) applied to TM (3.1) look as follows:

$$
\mathbf{T}(0)=\mathbf{I}, \quad \mathbf{T}(\pi)=\mathbf{P},
$$

where $\mathbf{I}$ is an identity operator, while $\mathbf{P}$ is a translational operator (3.5). Therefore, $\mathbf{P}$ may be diagonalized simultaneously with the rest of the family $\mathbf{T}(\theta)$. The matrix equation (3.4) in the diagonal representation reduces to the following equation for TM eigenvalues $\Lambda(\theta)$ and translation operator eigenvalue- $p$ :

$$
\Lambda(\pi-\theta)=p \Lambda^{*}(\theta), \quad p^{N}=1 .
$$

The second functional equation for $\Lambda(\theta)$ arises for TM with distorted boundary conditions, i.e. in the thermodynamic limit $(N \rightarrow \infty)$. Consider the product $\mathbf{T}^{\sim}(-\theta) \mathbf{T}(\theta)$ represented graphically in Fig. 6a where $\theta^{\prime}=\pi+\theta$. Substitute cyclic boundary conditions (3.2) by the following ones:

$$
\sigma_{N+1}=\mu_{N+1}=\sigma .
$$


The algebraic expression corresponding to the new variant of Fig. 6a reads:

$$
\begin{aligned}
{\left[\mathbf{T}^{\sim}(-\theta) \mathbf{T}(\theta)\right]_{\{\mu, \sigma\}} } & =\sum_{\{\lambda\}}\left[K_{\sigma_{1} \lambda_{1}}(\theta) K_{\mu_{1} \lambda_{1}}(-\theta)\right]\left[K_{\sigma_{2} \lambda_{1}}(\pi-\theta) K_{\mu_{2} \lambda_{1}}(\pi+\theta)\right] \\
& \ldots \cdot\left[K_{\sigma_{N} \lambda_{N}}(\theta) K_{\mu_{N} \lambda_{N}}(-\theta)\right]\left[K_{\sigma \lambda_{N}}(\pi-\theta) K_{\sigma \lambda_{N}}(\pi+\theta)\right] .
\end{aligned}
$$

Applying UC (2.22), (2.21) to the every factor in square brackets successively and beginning from the end of the product (4.3) one comes to the following result:

$$
\left[\mathbf{T}^{\sim}(-\theta) \mathbf{T}(\theta)\right]_{\{\mu, \sigma\}}=[f(\theta) g(\theta)]^{N} \delta_{\mu_{1}, \sigma_{1}} \ldots \delta_{\mu_{N}, \sigma_{N}} .
$$

The coincidence of $\mathbf{T}^{\sim}(-\theta) \mathbf{T}(\theta)(4.4)$ and the corresponding operator with the cyclic boundary conditions (3.2) in the thermodynamic limit $(N \rightarrow \infty)$ seems to be a reasonable conjecture assumed further. Thus,

$$
\mathbf{T}^{\sim}(-\theta) \mathbf{T}(\theta)=[f(\theta) g(\theta)]^{N} \mathbf{I} .
$$

In the diagonal representation Eq. (4.5) leads to the following equation for the eigenvalues:

$$
\Lambda^{*}(-\theta) \Lambda(\theta)=[f(\theta) g(\theta)]^{N} .
$$

As it was stated earlier, in the initial representation TM is positive when $0<\theta<\pi$. Thus, the Perron theorem is applicable when $\theta$ is inside the above interval [25, Chap. XIII, Sect. 2]. In accordance with this theorem, TM must have a real positive nondegenerate eigenvalue $\Lambda_{m}(\theta)$ exceeding the modulus of any other eigenvalue. The corresponding eigenvector, the vacuum vector, ought to have positive components. Since $\Lambda_{m}(\theta)$ and $\Lambda_{m}(\pi-\theta)$ are real and positive $(0<\theta<\pi)$, Eq. (4.2) fixes $p=1$. In other words, the vacuum vector is translationally invariant.

The partition function (3.3) in the thermodynamic limit is determined by the vacuum eigenvalue in accordance with the formula

$$
\mathbb{Z}=\left[\Lambda_{m}(\theta)\right]^{2 M} \text {. }
$$

It is helpful to introduce the density of the free energy per site of the lattice:

$$
-\beta F(\theta)=\frac{1}{2 M N} \log \mathbb{Z}(\theta)=\frac{1}{N} \log \Lambda_{m}(\theta) \equiv \log \zeta(\theta) .
$$

Thus introduced the real and positive quantity $\zeta(\theta)$ obeys two functional equations resulting from Eqs. (4.2), (4.6) and the translational invariance of the vacuum:

$$
\begin{aligned}
\zeta(\pi-\theta) & =\zeta(\theta), \\
\zeta(-\theta) \zeta(\theta) & =f(\theta) g(\theta) \equiv G(\theta) .
\end{aligned}
$$

These equations serve to evaluate the free energy (4.8).

\section{5. $Z_{n}$-Symmetry and Dual Transformation}

The $R$-matrix (2.10) remains unchanged after adding 1 modulo $n$ to each of its indices. One can speak of the $Z_{n}$-group as the group of $R$-matrix invariance.

However, we restrict ourselves with $R$-matrices posessing a higher symmetry: $Z_{n} \times Z_{n}$. Namely, we focus on $R_{\mu_{1} \mu_{2}}^{\sigma_{1} \sigma_{2}}(\theta)$ which is invariant after simultaneous shifting 
of only upper indices or only lower ones. That means

$$
R_{\mu_{1} \mu_{2}}^{\sigma_{1} \sigma_{2}}(\theta)=R_{\mu_{1}-\mu_{2}}^{\sigma_{1}-\sigma_{2}}(\theta)
$$

Factorized and $Z_{n}$-symmetric $R$-matrices (2.17) are forced to posess $Z_{n} \times Z_{n}$ invariance (5.1) equivalent to the $Z_{n}$ symmetry of the constituent factors:

$$
K_{\sigma_{1} \sigma_{2}}(\theta)=K_{\sigma_{1}-\sigma_{2}}(\theta) \text {. }
$$

The symmetries (2.11) and (2.12) in the new notations (5.1), (5.2) acquire the following form:

$$
\begin{aligned}
& R_{\mu}^{\sigma}(\theta)=R_{\mu}^{-\sigma}(\theta)=R_{-\mu}^{\sigma}(\theta), \\
& K_{\sigma}(\theta)=K_{-\sigma}(\theta) .
\end{aligned}
$$

We define the dual transformation (DT) of SW (5.1) by the formula

$$
\begin{aligned}
R_{\beta}^{\sim \alpha}(\theta) & =\frac{1}{n} \sum_{\sigma, \mu=0}^{n-1} \omega^{\alpha \mu+\beta \sigma} R_{\mu}^{\sigma}(\theta)=\frac{1}{n} \sum_{\sigma, \mu=0}^{n-1} \omega^{\alpha \sigma+\beta \mu} R_{\mu}^{\sigma}(\pi-\theta), \\
\omega & =\exp \left(\frac{2 \pi i}{n}\right) .
\end{aligned}
$$

The corresponding DT for $K_{\sigma}(\theta)$ agrees with (5.5):

$$
K_{\alpha}^{\sim}(\theta)=\frac{1}{n^{1 / 2}} \sum_{\sigma=0}^{n-1} \omega^{\alpha \sigma} K_{\sigma}(\pi-\theta)
$$

Reciprocal relations expressing $R_{\mu}^{\sigma}(\theta)$ via $R_{\mu}^{\sim \sigma}(\theta)$ and $K_{\sigma}(\theta)$ via $K_{\sigma}^{\sim}(\theta)$ are of the same form as (5.5) and (5.7), respectively, for the well-known formulas of the inverse Fourier transform are accompanied by symmetries (5.3) and (5.4).

Provided the solution $R_{\mu}^{\sigma}(\theta)$ of ET (2.14) or the solution $K_{\sigma}(\theta)$ of STR (2.18) is found, the DT (5.5) and (5.7), respectively, convert the known solutions into the new ones.

Just the following statements are valid:

Theorem 1. DT (5.5) of $Z_{n} \times Z_{n}$-symmetric $R$-matrices (5.1) leaves ET (2.14), the crossing condition (2.13), SBC (2.15), and UC (2.16) invariant once the above conditions are satisfied.

Theorem 2. Let $K_{\sigma}(\theta)$ obey STR (2.18), SBC (2.20), and UC (2.21), (2.22). Then dual $\mathrm{SWK}_{\sigma}^{\sim}(\theta)$ obeys the same constraints where the following substitutions should be produced:

$$
\begin{aligned}
\lambda^{\sim}\left(\theta_{1}, \theta_{2}, \theta_{3}\right) & =n \lambda^{-1}\left(\theta_{1}, \theta_{2}, \theta_{3}\right), \quad v^{\sim}=n^{1 / 2} v^{-1}, \\
f^{\sim}(\theta) & =n g(\theta), \quad g^{\sim}(\theta)=n^{-1} f(\theta) .
\end{aligned}
$$

One may conclude from two last formulas (5.8) that DT produces no change in the functional equations (4.9), (4.10).

Both theorems are easily proved by the direct substitution of (5.5) and (5.7) into the formulas enlisted in Theorems 1 and 2, respectively. The calculations are based on the properties of the Fourier transform and essentially on the symmetries (5.3) and (5.4), respectively. 
The above theorems allow the existence of the self-dual solutions of ET (2.14) and STR (2.18):

$$
\begin{gathered}
R_{\mu}^{\sim \sigma}(\theta)=R_{\mu}^{\sigma}(\theta), \\
K_{\sigma}^{\sim}(\theta)=\chi(\theta) K_{\sigma}(\theta), \quad \chi(\theta) \chi(\pi-\theta)=1,
\end{gathered}
$$

where the function in Eq. (5.9b) $\chi(\theta)$ depends on the normalization of $K_{\sigma}(\theta)$.

Physically, DT is a mapping of the initial spin system onto the dual one. Dual spins are situated at the sites of the dual lattice and their interaction is described by transformed SW (5.7) [26-28]. The system described by SW $R_{\mu}^{\sigma}(\theta)$ contains two sublattices dual to each other $[13,14,29,30]$. Spins $\sigma$ and $\mu$ exchange their sublattices after DT (5.5).

In addition to the full DT (5.5) we introduce a partial DT $R_{\mu}^{\sigma}(\theta)$ :

where

$$
K_{\mathbf{a}}(\theta)=\frac{1}{n^{1 / 2}} \sum_{\mu=0}^{n-1} \omega^{\alpha_{1} \mu} R_{\mu}^{\alpha_{2}}(\theta)
$$

$$
\mathbf{a}=\left(\alpha_{1}, \alpha_{2}\right) .
$$

The above transform moves spins $\mu$ from their sublattice to that of $\sigma$. Thus, SW $K_{\mathbf{a}}(\theta)$ describes a system containing two spins in every site of the lattice. Vector index a (5.11) takes its values in the group $Z_{n} \times Z_{n}$.

All the results hitherto obtained for SW $K_{\sigma}(\theta)$ may be naturally generalized for $K_{\mathbf{a}}(\theta)$ with vector index (5.11). Namely, any scalar index in the relations (2.18), (2.20)-(2.22), (3.1), (5.2), (5.7) should be substituted by a vector one. The addition of indices must be accomplished componentwise, and the summation over the vector index (5.11) is realized as the summation over $\alpha_{1}$ and $\alpha_{2}$ separately from 0 to $n-1$. Symbol $\delta_{\mathbf{a}, \mathbf{b}}$ designates the product $\delta_{\alpha_{1}, \beta_{1}} \cdot \delta_{\alpha_{2}, \beta_{2}}$. The product $\alpha \sigma$ in (5.7) should be changed to a scalar product as $=\alpha_{1} \sigma_{2}+\alpha_{2} \sigma_{2}$. If $n$ appears in some formula it must be substituted by $n^{2}$ except the expression (5.6) for $\omega$ which remains invariant. Instead of the symmetry (5.4) $K_{\mathbf{a}}(\theta)$ are supposed to obey the following constraints:

$$
K_{\mathbf{a}}(\theta) \equiv K_{\alpha_{1}, \alpha_{2}}(\theta)=K_{\alpha_{1},-\alpha_{2}}(\theta)=K_{-\alpha_{1}, \alpha_{2}}(\theta) .
$$

However, vector index STR (2.18) posess some specific symmetries that may be formulated as linear transformations of the vector index (5.11). Let us define two elements $A$ and $B$ generating a modular group [31, Sect. 13.22]:

$$
A \mathbf{a}=\left(\alpha_{2},-\alpha_{1}\right), \quad B \mathbf{a}=\left(\alpha_{1}, \alpha_{1}+\alpha_{2}\right) .
$$

STR (2.18) remain invariant after substitution

$$
K_{\mathbf{a}}(\theta) \rightarrow K_{E \mathbf{a}}(\theta),
$$

where $E$ is an arbitrary element of the modular group. Since any $E$ is nondegenerate it cannot alter SBC (2.20). As Ea takes the same set of the values as a, the partition function is an invariant of the modular group.

If $K_{\mathbf{a}}(\theta)$ is determined by Eq. (5.10) where the $R$-matrix obeys Eqs. (2.13) and (5.3), SW $K_{\mathbf{a}}^{\sim}(\theta)$ dual to $K_{\mathbf{a}}(\theta)$ are connected by transformation $A(5.13)$ :

$$
K_{\mathbf{a}}^{\sim}(\theta)=K_{A \mathbf{a}}(\theta) \text {. }
$$

For $K_{\mathrm{a}}(\theta)$ defined by Eq. (5.10) the following theorem is true. 
Theorem 3. If $R_{\mu}^{\sigma}(\theta)$ obey ET (2.14), crossing conditions (2.13), SBC (2.15) and the symmetry (5.3), then $K_{\mathrm{a}}(\theta)$ connected with $R_{\mu}^{\sigma}(\theta)$ by (5.10) obey STR (2.18) with $\lambda\left(\theta_{1}, \theta_{2}, \theta_{3}\right)=n, \operatorname{SBC}(2.20)$ with $v=n^{1 / 2}$ and symmetry relations (5.12). Certainly $R_{\mu}^{\sigma}(\theta)$ satisfy UC (2.16) with some $F(\theta)$; this fact results for $K_{\mathbf{a}}(\theta)(5.10)$ in UC (2.21), (2.22) with

$$
f(\theta)=n F(\theta), \quad g(\theta)=n^{-1} F(\theta) .
$$

Functional equations for evaluating the free energy of the system have the form (4.9), (4.10) with $G(\theta)=F^{2}(\theta)$. Note that the maximal eigenvalue of Baxter-type TM [13] obeys functional relations (4.9), (4.10) $[15,19]$ with $G(\theta)=F(\theta)$. This fact may be easily seen using the considerations analogous to that of Sect. 4 .

\section{Transfer Matrix and Quantum Commuting Hamiltonians ${ }^{1}$}

The TM of $Z_{n}$-symmetric systems may be expressed in terms of shift and multiplying operators [32-34]. We introduce them in the following way. Consider the vector of the spin configuration $\Phi_{\{\sigma\}}$ denoted by $\left|\sigma_{1}, \sigma_{2}, \ldots, \sigma_{N}\right\rangle$. The diagonal operator of multiplication for the variable at the $k^{\text {th }}$ site of the chain is defined according to the formula

$$
s_{k}\left|\sigma_{1}, \ldots, \sigma_{k}, \ldots, \sigma_{N}\right\rangle=\omega^{\sigma_{k}}\left|\sigma_{1}, \ldots, \sigma_{k}, \ldots, \sigma_{N}\right\rangle,
$$

while the shift operator $t_{k}$ is in conformity with the following one:

$$
t_{k}\left|\sigma_{1}, \ldots, \sigma_{k}, \ldots, \sigma_{N}\right\rangle=\left|\sigma_{1}, \ldots, \sigma_{k}+1, \ldots, \sigma_{N}\right\rangle,
$$

where addition is performed modulo $n$ as previously and the value of $\omega$ is given by (5.6).

The immediate corollaries from the definitions (6.1), (6.2), and (5.6) are

$$
s_{k}^{n}=t_{k}^{n}=s_{k} s_{k}^{+}=t_{k} t_{k}^{+}=1 .
$$

Any operators assigned to the different sites of the chain commute with each other, while $s_{k}$ and $t_{k}$ do not commute. The commutation relations between the latter may be easily obtained from (6.1), (6.2):

$$
s_{k} t_{k}=\omega t_{k} s_{k}, \quad s_{k} t_{k}^{+}=\omega^{*} t_{k}^{+} s_{k} .
$$

The expression (3.1) for TM provided $Z_{n}$ symmetry (5.2) is imposed takes the form

$$
\mathbf{T}_{\{\mu, \sigma\}}(\theta)=\prod_{k=1}^{N} K_{\mu_{k}-\sigma_{k}}(\theta) K_{\mu_{k}-\sigma_{k+1}}(\pi-\theta),
$$

which can be represented in terms of the above operators by introducing the operator functions

and

$$
P_{k}(\theta)=\sum_{\alpha=0}^{n-1} K_{\alpha}(\theta) t_{k}^{\alpha},
$$

$$
Q_{k+1 / 2}(\theta)=n^{-1 / 2} \sum_{\alpha=0}^{n-1} K_{\alpha}^{\sim}(\theta)\left(s_{k}^{+} s_{k+1}\right)^{\alpha},
$$

1 This approach is widely used in $[10,20,35]$. Nevertheless, Sect. 6 may be omitted without prejudice to further understanding 
where $K_{\alpha}^{\sim}(\theta)$ are the SW dual to $K_{\alpha}(\theta)(5.7)$. The operators (6.6) and (6.7) have the following matrix elements:

and

$$
\left\langle\mu_{1}, \ldots, \mu_{k}, \ldots, \mu_{N}\left|P_{k}(\theta)\right| \sigma_{1}, \ldots, \sigma_{k}, \ldots, \sigma_{N}\right\rangle=K_{\mu_{k}-\sigma_{k}}(\theta) \prod_{i \neq k} \delta_{\sigma_{i}, \mu_{i}},
$$

$$
\begin{aligned}
& \left\langle\mu_{1}, \ldots, \mu_{k}, \mu_{k+1}, \ldots, \mu_{N}\left|Q_{k+1 / 2}(\theta)\right| \sigma_{1}, \ldots, \sigma_{k}, \sigma_{k+1}, \ldots, \sigma_{N}\right\rangle \\
& \quad=n^{-1 / 2} \sum_{\alpha=0}^{n-1} K_{\alpha}^{\sim}(\theta) \omega^{\alpha\left(\sigma_{k+1}-\mu_{k}\right)} \prod_{i=1}^{N} \delta_{\sigma_{l}, \mu_{l}}=K_{\mu_{k}-\sigma_{k+1}}(\pi-\theta) \prod_{i=1}^{N} \delta_{\sigma_{i}, \mu_{i}}
\end{aligned}
$$

The latter equality in (6.9) was obtained with the help of the formula reciprocal to (5.7).

The cyclic boundary conditions (3.2) require the corresponding projection operators to be introduced on the auxilary chain containing $N+1$ spins :

$$
\mathfrak{p}=\sum_{\alpha=0}^{n-1} t_{N+1}^{\alpha}, \quad \mathfrak{q}=n^{-1} \sum_{\alpha=0}^{n-1}\left(s_{1}^{+} s_{N+1}\right)^{\alpha} .
$$

It may be readily verified using Eqs. (6.5)-(6.10) that matrix elements of the operators $\mathbf{T}(\theta)$ and $\mathfrak{p} \mathfrak{I}(\theta) \mathfrak{q}$ coincide :

$$
\left\langle\mu_{1}, \ldots, \mu_{N}, \mu_{N+1}|\mathfrak{p} \mathfrak{I}(\theta) \mathfrak{q}| \sigma_{1}, \ldots, \sigma_{N}, \sigma_{N+1}\right\rangle=\delta_{\sigma_{1}, \sigma_{N+1}} \mathbf{T}_{\{\mu, \sigma\}}(\theta),
$$

where

$$
\mathfrak{I}(\theta)=Q_{N+1 / 2}(\theta) P_{N}(\theta) Q_{N-1 / 2}(\theta) P_{N-1}(\theta) \ldots Q_{3 / 2}(\theta) P_{1}(\theta)
$$

TM in terms of operators (6.1), (6.2) have been examined in [20]. They were demonstrated to be generating functionals for a set of quantum commuting Hamiltonians. Consider a Taylor series for operator $\mathfrak{I}(\theta)$ in the vicinity of $\theta=0$. Due to SBC (2.20)

$$
P_{k}(0)=v \mathbf{I}, \quad Q_{k+1 / 2}(0)=v^{-1} \mathbf{I},
$$

therefore,

$$
\mathfrak{I}(0)=\mathbf{I}
$$

The next term of the Taylor expansion of (6.12) is linear in $\theta$ with the operator coefficient

$$
\mathscr{H}=v^{-1} \sum_{k=1}^{N} P_{k}^{\prime}(0)+v \sum_{k=1}^{N} Q_{k+1 / 2}^{\prime}(0) .
$$

Here the prime denotes the derivative with respect to $\theta$.

Thus,

$$
\mathfrak{I}(\theta)=\mathbf{I}+\mathscr{H} \theta+\sum_{n=2}^{\infty} \mathscr{T}_{n} \theta^{n} .
$$

One cannot surely state whether the expansion (6.15) is convergent. Yet formally all the coefficients of the Taylor series (6.15) must commute in the limit $N \rightarrow \infty$. Their commutativity is a straightforward corollary of Eq. (3.7). Really the boundary conditions imposed by operators (6.10) become inessential in the limit $N \rightarrow \infty$. So the operators themselves may be neglected. 
However, $\mathscr{T}_{n}$ obtained from (6.15) are not local. The standard way to construct local motion integrals is to rearrange the series (6.15) in the form:

$$
\log \mathfrak{T}(\theta)=\sum_{n=1}^{\infty} \mathscr{C}_{n} \theta^{n}, \quad \mathscr{C}_{1}=\mathscr{H} .
$$

The quantities $\mathscr{C}_{n}$ represent a set of quantum quasi-local Hamiltonians [20, 35]. For $n \geqq 2 \mathscr{C}_{n}$ are motion integrals with respect to the operator $\mathscr{H}(6.14)$ [20].

We finish the present section describing DT in terms of TM (6.11). As in $[33,34]$, we introduce dual operators $t_{k+1 / 2}^{\sim}$ and $s_{k+1 / 2}^{\sim}$ related with $t_{k}$ and $s_{k}$ as follows :

$$
t_{k}=\tilde{s_{k+1 / 2}} \tilde{s_{k-1 / 2}^{++}}, \quad t_{k+1 / 2}^{\sim}=s_{k+1}^{\sim} s_{k}^{+}, \quad 1 \leqq k \leqq N, \quad t_{1 / 2}^{\sim}=s_{N+1}^{+} s_{1} .
$$

Dual operators form the algebra isomorphic to that of $t_{k}$ and $s_{k}(6.4)$ with the substitution $t_{k} \rightarrow t_{k+1 / 2}^{\sim}, s_{k} \rightarrow \tilde{s_{k+1 / 2}}$. The operator functions (6.6), (6.7) after the change of variables (6.17) may be rewritten as

$$
\begin{aligned}
P_{k}(\theta) & =\sum_{\alpha=0}^{n-1} K_{\alpha}(\theta) t_{k}^{\alpha}=\sum_{\alpha=0}^{n-1} K_{\alpha}(\theta)\left(s_{k-1 / 2}^{\sim+} s_{k+1 / 2}\right)^{\alpha} \equiv n^{1 / 2} Q_{k}^{\sim}(\theta) \\
Q_{k+1 / 2}(\theta) & =n^{-1 / 2} \sum_{\alpha=0}^{n-1} K_{\alpha}^{\sim}(\theta)\left(s_{k}^{+} s_{k+1}\right)^{\alpha} \\
& =n^{-1 / 2} \sum_{\alpha=0}^{n-1} K_{\alpha}^{\sim}(\theta) t_{k+1 / 2}^{\sim \alpha} \equiv n^{-1 / 2} P_{k+1 / 2}^{\sim}(\theta) .
\end{aligned}
$$

$\operatorname{TM} \mathfrak{I}(\theta)(6.12)$ in terms of dual $Q_{k}^{\sim}$ and $P_{k+1 / 2}^{\sim}$ is rather similar to that in terms of initial $P_{k}$ and $Q_{k+1 / 2}$ :

$$
\mathfrak{I}(\theta)=P_{N+1 / 2}^{\sim}(\theta) Q_{N}^{\sim}(\theta) P_{N-1 / 2}^{\sim}(\theta) Q_{N-1}^{\sim}(\theta) \ldots P_{3 / 2}^{\sim}(\theta) Q_{1}^{\sim}(\theta),
$$

and

$$
\mathfrak{q}=n^{-1} \sum_{\alpha=0}^{n-1} t_{1 / 2}^{\sim \alpha}=n^{-1} \mathfrak{p}^{\sim}, \quad \mathfrak{p}=\sum_{\alpha=0}^{n-1}\left(s_{N+1 / 2}^{\tilde{\alpha}} \tilde{s}_{1 / 2}^{\sim}\right)^{\alpha}=n \mathfrak{q}^{\sim} .
$$

Here the last equality is valid only in the invariant subspace defined by a projection operator

$$
\mathfrak{P}=n^{-1} \sum_{\alpha=0}^{n-1}\left(\prod_{k=1}^{N+1} t_{k}\right)^{\alpha}
$$

In the thermodynamic limit DT reduces to the interchange

$$
K_{\alpha}(\theta) \leftrightarrow K_{\alpha}^{\sim}(\theta)
$$

\section{STR in the $n$-State Potts Models}

The following simplest ansatz for SW (5.2) when $0 \leqq \alpha \leqq n-1$ is known as the case of the $n$-state Potts model [11]:

$$
K_{\alpha}(\theta)=1+v(\theta) \delta_{\alpha, 0} .
$$


The Ising model is included in this class $(n=2)$. STR (2.18) accounting for (7.1) reduce to a set of functional equations for the function $v(\theta)$ :

$$
\begin{gathered}
n+v\left(\theta_{1}\right)+v\left(\theta_{2}\right)+v\left(\theta_{3}\right)=\lambda\left(\theta_{1}, \theta_{2}, \theta_{3}\right), \\
v\left(\theta_{i}\right) v\left(\theta_{j}\right)=\lambda\left(\theta_{1}, \theta_{2}, \theta_{3}\right) v\left(\pi-\theta_{k}\right), \\
v\left(\theta_{1}\right) v\left(\theta_{2}\right) v\left(\theta_{3}\right)=\lambda\left(\theta_{1}, \theta_{2}, \theta_{3}\right)\left[v\left(\pi-\theta_{1}\right) v\left(\pi-\theta_{2}\right) v\left(\pi-\theta_{3}\right)\right. \\
\left.+v\left(\pi-\theta_{1}\right) v\left(\pi-\theta_{2}\right)+v\left(\pi-\theta_{2}\right) v\left(\pi-\theta_{3}\right)+v\left(\pi-\theta_{3}\right) v\left(\pi-\theta_{1}\right)\right] .
\end{gathered}
$$

Here $(i, j, k)$ is an arbitrary permutation of $(1,2,3)$. For $n=2$ only two linear combinations of Eqs. (7.2)-(7.4) are independent. This case will be considered in the next section.

Note that $\operatorname{SBC}(2.20)$ in terms of the function $v(\theta)$ are singular:

$$
\lim _{\theta \rightarrow 0} v(\theta)=\infty, \quad v(\pi)=0 .
$$

The solution of Eqs. (7.2)-(7.4) is given in Appendix A. It is helpful to introduce a parameter $\mu$ instead of $n$ by the formula (A.10). For $n>4 \mu$ is real, for $n=4 \mu=0$, for $n<4 \mu$ is pure imaginary. Namely, for $n=3 \mu=i / 6$ and for $n=2 \mu=i / 4$ Quoting (A.11) we write

$$
v(\theta)=2 \cosh \mu \pi \sinh \mu(\pi-\theta) / \sinh \mu \theta .
$$

Parametrization (7.6) was obtained in [36].

One may perform DT (5.7) using (7.1), (7.6):

$$
K_{\alpha}^{\sim}(\theta)=n^{1 / 2} \delta_{\alpha, 0}+n^{-1 / 2} v(\pi-\theta)=[\sinh \mu \theta / \sinh \mu(\pi-\theta)] K_{\alpha}(\theta) .
$$

The relation (7.7) agrees with the definition (5.9b), so $K_{\alpha}(\theta)$ is self-dual. In other words, the solution involved describes the critical point of the Potts model in agreement with the previous results of the Potts models' integrability $[10,20,36-$ 39]. The quantum Hamiltonian and the involutory conservation laws were examined in $[10,20]$.

The straightforward substitution of (7.1), (7.6) into UC (2.21), (2.22) gives the functions on the right-hand side of $\mathrm{UC}$ :

$$
f(\theta)=v(\theta) v(-\theta) ; \quad g(\theta)=1 .
$$

The function $G(\theta)$ on the right-hand side of (4.10) is the product of $f(\theta)$ and $g(\theta)$ :

$$
\zeta(\theta) \zeta(-\theta)=v(\theta) v(-\theta)=-n \frac{\sinh \mu(\pi-\theta) \sinh \mu(\pi+\theta)}{\sinh ^{2} \mu \theta} .
$$

The system (4.9), (7.9) will be solved in Sect. 10.

\section{The Ising Model}

The Ising model is a particular case of Potts models $(n=2)$ as it was mentioned in the previous section. However, the number of independent STR in this peculiar case is less than in the general one. So the class of permissible solutions is wider. The Ising model is integrable outside its critical point [1]. 
According to the conventions assumed in Sect. $7 K_{1}(\theta)=1$. The model is thus characterized by a single function $K_{0}(\theta)=x(\theta)$. STR (2.18) read:

$$
\begin{aligned}
1+x\left(\theta_{1}\right) x\left(\theta_{2}\right) x\left(\theta_{3}\right) & =\lambda\left(\theta_{1}, \theta_{2}, \theta_{3}\right) x\left(\pi-\theta_{1}\right) x\left(\pi-\theta_{2}\right) x\left(\pi-\theta_{3}\right), \\
x\left(\theta_{i}\right)+x\left(\theta_{j}\right) x\left(\theta_{k}\right) & =\lambda\left(\theta_{1}, \theta_{2}, \theta_{3}\right) x\left(\pi-\theta_{i}\right),
\end{aligned}
$$

where $(i, j, k)$ is an arbitrary permutation of $(1,2,3)$.

Equations (8.1), (8.2) are solved in Appendix B. We quote formula (B.13)

$$
x(\theta)=k_{1}^{-1 / 2} \operatorname{cs}\left(\frac{K \theta}{2 \pi} \mid k\right)=\frac{\vartheta_{2}\left(\frac{\theta}{4} \mid \tau\right)}{\vartheta_{1}\left(\frac{\theta}{4} \mid \tau\right)} .
$$

Here $k_{1}$ and $k$ are complementary moduli of elliptic functions (see [31, Sects. 13.9, $13.19,13.20])^{2}$. Perform DT (5.7):

$$
\begin{aligned}
& K_{0}^{\sim}(\theta)=2^{-1 / 2}[x(\pi-\theta)+1]=\exp \left(-i \frac{\pi}{8}\right) \frac{\vartheta_{2}\left(\frac{\theta}{4} \mid \tau+1 / 2\right)}{\vartheta_{1}\left(\frac{\pi-\theta}{4} \mid \tau\right)}, \\
& K_{1}^{\sim}(\theta)=2^{-1 / 2}[x(\pi-\theta)-1]=\exp \left(-i \frac{\pi}{8}\right) \frac{\vartheta_{1}\left(\frac{\theta}{4} \mid \tau+1 / 2\right)}{\vartheta_{1}\left(\frac{\pi-\theta}{4} \mid \tau\right)} .
\end{aligned}
$$

DT (8.4), (8.5) may be represented in the form analogous to (5.9b)

$$
K_{\alpha}^{\sim}(\theta \mid \tau)=g(\theta) K_{\alpha}(\theta \mid \tau+1 / 2)
$$

where $g(\theta)=K_{1}^{\sim}(\theta)$ in $(8.5)$.

Let us elucidate the relations between the elliptic parameters $\theta, \tau$ and the physical parameters of the Ising model $\beta_{i}=J_{i} / T$ with $T$ denoting temperature and $J_{1,2}$ denoting interaction constants. Since $K_{1}(\theta) \equiv 1$ (see Fig. 5), one may conclude :

$$
\exp \left(2 \beta_{1}\right)=x(\theta), \quad \exp \left(2 \beta_{2}\right)=x(\pi-\theta) \equiv y(\theta) .
$$

Here the dependence of $x(\theta)$ and $y(\theta)$ on $\tau$ is implicit.

Excluding $\theta$ from Eq. (8.7) one may express $\tau$ via $\beta_{1}$ and $\beta_{2}$ directly. For this purpose combine the formulas (B.10) and (B.12):

$$
\sinh \left(2 \beta_{1}\right) \sinh \left(2 \beta_{2}\right)=\frac{\left[x^{2}(\theta)-1\right]\left[y^{2}(\theta)-1\right]}{4 x(\theta) y(\theta)}=\frac{1+k_{1}}{2 k_{1}^{1 / 2}} .
$$

The quantity on the right-hand side of (8.8) may be expressed via $\tau$ in a direct way in accordance with [31, Sects. 13.20, 13.23]:

$$
\sinh \left(2 \beta_{1}\right) \cdot \sinh \left(2 \beta_{2}\right)=\frac{\vartheta_{3}^{2}(0 \mid 2 \tau)}{\vartheta_{4}^{2}(0 \mid 2 \tau)} .
$$

2 Note that we use theta functions that differ from those defined in [31, Sect. 13.19] by multiplication of their argument by a factor $\pi$ 
The relationship (8.9) is simplified in the vicinity of the critical point. The latter is fixed by the Kramers-Wannier condition [26]:

$$
\sinh \left(2 \beta_{1}^{c}\right) \cdot \sinh \left(2 \beta_{2}^{c}\right)=1 .
$$

Therefore, for $T$ sufficiently close to $T_{c}$.

$$
\sinh \left(2 \beta_{1}\right) \cdot \sinh \left(2 \beta_{2}\right)-1=\frac{T_{c}-T}{T_{c}}\left[2 \beta_{1}^{c} \operatorname{coth} 2 \beta_{1}^{c}+2 \beta_{2}^{c} \operatorname{coth} 2 \beta_{2}^{c}\right],
$$

while (8.9) gives [31, Sect. 13.23]:

$$
\sinh \left(2 \beta_{1}\right) \cdot \sinh \left(2 \beta_{2}\right)-1=2 \frac{\vartheta_{2}^{2}(0 \mid 4 \tau)}{\vartheta_{4}^{2}(0 \mid 2 \tau)} .
$$

The right-hand side of (8.12) vanishes at $\tau \rightarrow i \infty$. This limit corresponds to the critical point. In the vicinity of the latter the right-hand side is exponential in $\tau$ [31, Sect. 13.19]. That allows us to express $\tau$ as a function of temperature in a transparent way:

$$
\tau=\frac{1}{2 \pi i} \log \frac{T_{c}-T}{T_{c}}+\frac{1}{2 \pi i} \log \frac{\beta_{1}^{c} \operatorname{coth} 2 \beta_{1}^{c}+\beta_{2}^{c} \operatorname{coth} 2 \beta_{2}^{c}}{4}+o(1) .
$$

In the vicinity of the transition point DT (8.6) is reduced to a reversal of the sign for $\left(T-T_{c}\right)$ [26]. The latter is equivalent to the shift of $\tau \rightarrow \tau+1 / 2$ in agreement with the general formula (8.6). Reversing the sign of $\left(T-T_{c}\right)$ twice, one shifts $\tau \rightarrow \tau+1$. However, SW (8.3) do not change under this transformation since the right-hand side of (8.3) is periodic in $\tau$ with a period equal to unity [31, Sect. 13.22].

SW (8.3) are double-periodic in $\theta$ with periods $4 \pi$ and $8 \pi \tau$ [31, Sect. 13.19]. The more subtle property worth attention is their quasi-periodicity analogous to that discussed in [23]:

$$
K_{\alpha}(\theta+2 \pi)=(-1)^{\alpha+1} x^{-1}(\theta) K_{\alpha+1}(\theta) ; \quad K_{\alpha}(\theta+4 \pi \tau)=(-1)^{\alpha+1} K_{\alpha}(\theta) .
$$

The correctness of (8.14) may be checked directly with the aid of (8.3) and [31, Sect. 13.19, Table 8].

The functions $f(\theta)$ and $g(\theta)$ in UC (2.21), (2.22) obviously are

$$
f(\theta)=1-x^{2}(\theta), \quad g(\theta)=1 .
$$

Recall that $x(\theta)$ is odd. Rewrite $f(\theta)$ in a more suitable form [31, Sects. 13.23, 13.19]:

$$
f(\theta)=1-x^{2}(\theta)=1-\frac{\vartheta_{2}^{2}\left(\frac{\theta}{4} \mid \tau\right)}{\vartheta_{1}^{2}\left(\frac{\theta}{4} \mid \tau\right)}=-\frac{\vartheta_{2}^{2}(0 \mid \tau)}{\vartheta_{1}^{2}\left(\frac{\pi}{4} \mid \tau\right)} \frac{\vartheta_{1}\left(\frac{\pi-\theta}{4} \mid \tau\right) \vartheta_{1}\left(\frac{\pi+\theta}{4} \mid \tau\right)}{\vartheta_{1}^{2}\left(\frac{\theta}{4} \mid \tau\right)} .
$$

This function should be inserted instead of $G(\theta)$ in Eq. (4.10) since $G(\theta)=f(\theta) g(\theta)$. The evaluation of $\zeta(\theta)$ based on Eqs. (4.9), (4.10), and (8.16) is performed in Sect. 10. 


\section{STR in the Ashkin-Teller Model}

The Ashkin-Teller model [40] is a spin model with $Z_{2} \times Z_{2}$ symmetry in conformity with the conventions of Sect. 5. The matrix of SW $K_{\alpha_{1} \alpha_{2}}(\theta)$ is represented by four quantities since either of the indices takes the values 0,1 separately.

The solution of STR for this model may be extracted from the known solution of ET in the Baxter model $[14,15]$ by virtue of Eq. (5.10) (Theorem 3, Sect. 5). An explicit calculation (C.9) is carried out in Appendix C.

A rather cumberous inspection of STR shows that all the solutions of STR for the Ashkin-Teller model are exhausted by (C.9) and the factorized ones:

$$
K_{\alpha_{1} \alpha_{2}}(\theta)=K_{\alpha_{1}}^{1}(\theta) K_{\alpha_{2}}^{2}(\theta)
$$

where either $K_{\alpha_{z}}^{i}(\theta)$ is some solution of the Ising model (8.3). The solutions obtained from the above ones by applying the symmetry (5.14) must be taken into consideration as well. Really, if some solution $K_{\mathbf{a}}(\theta)$ is found, any permutation of $K_{01}(\theta), K_{10}(\theta), K_{11}(\theta)$ leads to another solution of STR. STR (2.18), SBC (2.20), and UC (2.21), (2.22) remain unchanged after the above permutations.

The following renormalization of (C.9) is convenient for further developments:

$$
\begin{aligned}
K_{00}(\theta) & =\varrho_{1}(\theta) \frac{\vartheta_{1}(v(2 \pi-\theta) \mid 2 \tau)}{\vartheta_{1}(v \theta \mid 2 \tau)}, \quad K_{01}(\theta)=\varrho_{1}(\theta) \frac{\vartheta_{3}(v(2 \pi-\theta) \mid 2 \tau)}{\vartheta_{3}(v \theta \mid 2 \tau)} \\
K_{10}(\theta) & =\varrho_{1}(\theta) \frac{\vartheta_{4}(v(2 \pi-\theta) \mid 2 \tau)}{\vartheta_{4}(v \theta \mid 2 \tau)}, \quad K_{11}(\theta)=\varrho_{1}(\theta) \frac{\vartheta_{2}(v(2 \pi-\theta) \mid 2 \tau)}{\vartheta_{2}(v \theta \mid 2 \tau)} \\
\varrho_{1}(\theta) & =2^{3 / 2} \varrho \vartheta_{1}(v \theta \mid 2 \tau) \vartheta_{2}(v \theta \mid 2 \tau) \vartheta_{3}(v \theta \mid 2 \tau) \vartheta_{4}(v \theta \mid 2 \tau) \\
& =2^{1 / 2} \varrho \vartheta_{1}(2 v \theta \mid 2 \tau) \vartheta_{2}(0 \mid 2 \tau) \vartheta_{3}(0 \mid 2 \tau) \vartheta_{4}(0 \mid 2 \tau)
\end{aligned}
$$

Abstracting from the obvious variations of $\varrho_{1}(\theta)$ we find out that the above permutations are closely related to a group of modular transformations of $2 \tau$. Namely,

$$
\begin{aligned}
K_{\mathbf{a}}(\theta ; v \mid 2 \tau+1) & =K_{A \mathbf{a}}(\theta ; v \mid 2 \tau), \\
K_{\mathbf{a}}(\theta ; v \mid-1 / 2 \tau) & =\exp \left[i 8 \tau v^{2}(\pi-\theta)\right] K_{B \mathbf{a}}(\theta ; 2 v \tau \mid 2 \tau) .
\end{aligned}
$$

Here transformations $A$ and $B$ are defined by Eqs. (5.13). The notations coincide with that in [31, Sect. 13.22]. The relations $(9.3 \mathrm{a}, \mathrm{b})$ demonstrate the free energy of the Ashkin-Teller model to be changed by an inessential additive function after the modular transformation of $2 \tau$.

SW (9.1) exhibit quasi-periodicity analogous to (8.14) and that described in [23]. An easy examination of formulas (9.1) leads to the following symbolic equalities $^{3}$ (see [31, Sect. 13.19, Table 8]):

$$
\begin{gathered}
K_{\alpha \beta}(\theta+\pi / 2 v)=(-1)^{\alpha+\beta+1} K_{\alpha+1, \beta+1}(\theta), \\
K_{\alpha \beta}(\theta+\pi \tau / \nu)=(-1)^{\beta+1} \exp (2 \pi i v) K_{\alpha+1, \beta}(\theta) .
\end{gathered}
$$

3 We neglect trivial variations of $\varrho_{1}(\theta)$ in (9.4) 
We define the physical parameters $\kappa_{1}(\theta), \kappa_{2}(\theta), \kappa_{3}(\theta)$ of the Ashkin-Teller model by the formula

$$
K_{\alpha \beta}(\theta)=\exp \left[\kappa_{1}(\theta)(-1)^{\alpha}+\kappa_{2}(\theta)(-1)^{\beta}+\kappa_{3}(\theta)(-1)^{\alpha+\beta}\right] .
$$

The general anisotropic Ashkin-Teller model is described by six independent interactions. In the particular case under consideration these six quantities reduce to three $\kappa_{i}(\theta)$ and three $\kappa_{i}(\pi-\theta)(i=1,2,3)$. Certainly, since only three parameters describe the solution (9.1) adequately, the above six quantities are not independent.

Just as in the case of the Ising model one may express $\tau$ via $\kappa_{i}(\theta)$. For this purpose insert SW $R_{\alpha}^{\beta}(\theta)$ and dual SW $R_{\beta}^{\sim \alpha}(\theta)$ expressed via $K_{\mathbf{a}}(\theta)$ by virtue of Eqs. (5.5), (5.10) into (C.13) and use the definition (9.5). The result reads:

$$
\frac{\vartheta_{3}^{4}(0 \mid 2 \tau)}{\vartheta_{4}^{4}(0 \mid 2 \tau)}=\frac{\cosh 4 \kappa_{2}-\cosh 4 \kappa_{3}}{\cosh 4 \kappa_{1}-\cosh 4 \kappa_{3}} .
$$

This formula is considerably simplified, becoming explicit with respect to $\tau$ when $\tau \rightarrow i \infty\left(\right.$ or $\left.\kappa_{1} \rightarrow \kappa_{2}\right)$ :

$$
\tau=(1 / 2 \pi i) \log \left(\kappa_{2}-\kappa_{1}\right)+\text { const }+o\left(\kappa_{1}-\kappa_{2}\right) .
$$

Note the similarity of Eq. (9.7) and the analogous expression (8.13) in the case of the Ising model.

The self-dual region $\kappa_{1}=\kappa_{2}$ is of special interest since the symmetry of the Ashkin-Teller model here is increased from $Z_{2} \times Z_{2}$ to $Z_{4}$. Introduce a scalar variable $\sigma=0,1,2,3$ instead of a vector one $\mathbf{a}=(\alpha, \beta)$ by the rule

$$
\exp (i \pi \sigma / 2)=2^{-1 / 2}\{\exp [i \pi(\alpha-1 / 4)]+\exp [i \pi(\beta+1 / 4)]\} .
$$

Substitution of (9.8) into (9.5) gives the result

$$
\begin{aligned}
K_{\mathbf{a}-\mathbf{b}}(\theta) & =K_{\sigma-\mu}(\theta), \\
K_{\sigma}(\theta) & =\exp \left[2 \kappa_{1}(\theta) \cos (\pi \sigma / 2)+\kappa_{3}(\theta)(-1)^{\sigma}\right] .
\end{aligned}
$$

When $\kappa_{1} \neq \kappa_{2}$ SW $K_{\sigma \mu}(\theta)$ is not $Z_{4}$-invariant depending on its indices $\sigma$ and $\mu$ separately. The limit $\tau \rightarrow i \infty$ in (9.1) yields [31, Sect. 13.19] the following parametrization of the $Z_{4}$-symmetric solution:

$$
\frac{K_{1}(\theta)}{K_{0}(\theta)}=\frac{K_{3}(\theta)}{K_{0}(\theta)}=\frac{\sin v \theta}{\sin v(2 \pi-\theta)}, \quad \frac{K_{2}(\theta)}{K_{0}(\theta)}=\frac{\tan v \theta}{\tan v(2 \pi-\theta)} .
$$

To finish this section we derive the functional equations for the maximal eigenvalue of TM. It is readily seen from (9.1):

$$
g(\theta)=K_{\mathbf{a}}(\pi+\theta) K_{\mathbf{a}}(\pi-\theta)=\varrho_{1}(\pi-\theta) \varrho_{1}(\pi+\theta) .
$$

Now using the relationship between $f(\theta)$ and $g(\theta)$ (5.16) we obtain:

$$
f(0)=4 g(\theta)=-4 \varrho_{1}(\theta) \varrho_{1}(-\theta) \frac{\vartheta_{1}(2 v(\pi-\theta) \mid 2 \tau) \vartheta_{1}(2 v(\pi+\theta) \mid 2 \tau)}{\vartheta_{1}^{2}(2 v \theta \mid 2 \tau)} .
$$


Here the factors $\varrho_{1}(\theta)$ and $\varrho_{1}(-\theta)$ are extracted by virtue of the formula (9.2) for convenience in further calculations. After all, Eq. (4.10) reads:

$$
\begin{aligned}
\zeta(\theta) \zeta(-\theta)= & -4 \varrho_{1}(\theta) \varrho_{1}(-\theta) \varrho_{1}(\pi-\theta) \varrho_{1}(\pi+\theta) \\
& \cdot \frac{\vartheta_{1}(2 v(\pi-\theta) \mid 2 \tau) \vartheta_{1}(2 v(\pi+\theta) \mid 2 \tau)}{\vartheta_{1}^{2}(2 v \theta \mid 2 \tau)} .
\end{aligned}
$$

\section{Evaluation of the Partition Functions}

In this section we evaluate the partition functions of the models discussed previously. In fact, we solve the functional equations (4.9), (4.10) that rule the behaviour of the maximal TM eigenvalue as a function of the spectral parameter $\theta$. To solve these equations uniquely, we appeal to the quasi-periodicity properties of $\mathrm{TM}$ and its eigenvalues that will be discussed for specific models a little later. Recall the models under consideration: the critical Potts model (Sect. 7), the Ising model (Sect. 8), the Ashkin-Teller model (Sect. 9). The latter is described by a maximal number of parameters: $\theta, v, \tau$. Functional equations $(4.10)$ specified by Eq. (7.9) for the Potts models and by Eq. (8.16) for the Ising model coincide with that for the Ashkin-Teller model (9.13) when parameters $v$ and $\tau$ are fixed properly. The same may be stated as far as quasi-periodicity properties are concerned.

The Ashkin-Teller model (Sect. 9). SW (9.1) are real and positive for

$$
0<2 v \theta<\pi, \quad 0<2 v(2 \pi-\theta)<\pi .
$$

Quasi-periodicity of pair SW (9.4) causes the analogous properties of TM (3.1) of the Ashkin-Teller model

$$
\begin{aligned}
& \mathbf{T}(\theta+\pi / 2 \nu)=\mathbf{V}_{1} \mathbf{V}_{2} \mathbf{T}(\theta), \\
& \mathbf{T}(\theta+\pi \tau / \nu)=\mathbf{V}_{1} \mathbf{T}(\theta),
\end{aligned}
$$

where operators $\mathbf{V}_{1}$ and $\mathbf{V}_{2}$ are defined in the following way:

$$
\left(\mathbf{V}_{k}\right)_{\{\mathbf{a}, \mathbf{b}\}}=\prod_{i=1}^{N} \delta_{\mathbf{a}_{i}-\mathbf{b}_{i}, \mathbf{e}_{k}}, \quad k=1,2, \quad \mathbf{e}_{1}=(0,1), \quad \mathbf{e}_{2}=(1,0)
$$

The corollaries of the definition (10.4) are

$$
\mathbf{V}_{k}^{2}=\mathbf{I}, \quad \mathbf{V}_{k}^{+}=\mathbf{V}_{k}, \quad k=1,2 .
$$

If $\mathrm{TM}$ is normalized according to (4.1), $\mathbf{V}_{1}$ and $\mathbf{V}_{2}$ belong to the commutative family of TM:

$$
\mathbf{V}_{1}=\mathbf{T}(\pi \tau / \nu), \quad \mathbf{V}_{2}=\mathbf{T}(\pi / 2 \nu+\pi \tau / v)
$$

Therefore, both operators may be diagonalized simultaneously with the rest of the family. The eigenvalues of $\mathbf{V}_{k}$ due to Eq. (10.5) are equal to \pm 1 . Any eigenvector of $\mathbf{T}(\theta)$ with its eigenvalue may be thus ascribed to one of the four categories according to the corresponding eigenvalues of $\mathbf{V}_{1}$ and $\mathbf{V}_{2}$.

Quasi-periodicity (10.2), (10.3) causes the same quasi-periodicity of the total ensemble of eigenvalues for every of the above four categories separately. However, it does not mean quasi-periodicity for any given eigenvalue, since an arbitrary exchange of individual eigenvalues might occur after translation of $\theta$ by some quasi-period. 
The above transposition of eigenvalues might be expected to occur in the points where $\mathbf{T}(\theta)$ becomes degenerate, i.e. two or more of its eigenvalues become equal. By virtue of SBC (4.1) the points of degeneracy for $\mathbf{T}(\theta)$ are $\theta=0$ and $\theta=\pi$. Certainly, these points translated by any quasi-period are degeneracy points as well. Since Eq. (10.1) dictates $v<1 / 4$, any point in the interval $0<\theta<\pi$ translated by the quasi-period (10.2) $\pi / 2 v$ is sure to pass through some degeneracy point.

The requirement of quasi-periodicity (10.2) applied to the eigenvalues seems to be too severe for the above reasons. Just as nothing is known about degeneracy points besides the facts above mentioned, the following hypothesis seems to be reasonable:

Hypothesis. The eigenvalues of $T M-\Lambda(\theta)$ have a quasi-period $\pi \tau / v$ :

$$
\Lambda(\theta+\pi \tau / v)= \pm \Lambda(\theta) \text {. }
$$

The maximal eigenvalue $\Lambda_{m}(\theta)$ is periodic (10.3) [15]. The free energy (4.8) being its logarithm has also a period $\pi \tau / v$ :

$$
\log \zeta(\theta+\pi \tau / \nu)=\log \zeta(\theta)
$$

Note the last assumption (10.8) is more restricting than that of periodicity for $\zeta(\theta)$.

Rewrite Eq. (9.13) using the Jacobi modular transformation [31, Sect. 13.22]:

$$
\begin{gathered}
\zeta(-\theta) \zeta(\theta)=-4 \varrho_{1}(\theta) \varrho_{1}(-\theta) \varrho_{1}(\pi-\theta) \varrho_{1}(\pi+\theta) \exp \left(-i 4 \pi v^{2} / \tau\right) \\
\vartheta_{1}(v(\pi-\theta) / \tau \mid-1 / 2 \tau) \vartheta_{1}(v(\pi+\theta) / \tau \mid-1 / 2 \tau) \vartheta_{1}^{-2}(v \theta / \tau \mid-1 / 2 \tau) .
\end{gathered}
$$

Substitute for the unknown function the following one:

$$
\zeta(\theta)=2 \varrho_{1}(\theta) \varrho_{1}(\pi-\theta) \exp \left(-i 2 \pi v^{2} / \tau\right) \frac{\vartheta_{1}(v(\pi-\theta) / \tau \mid-1 / 2 \tau)}{\vartheta_{1}(v \theta / \tau \mid-1 / 2 \tau)} \varphi(\theta) .
$$

Since $\log \zeta(\theta)$ is assumed periodic, it may be expanded into a Fourier series:

$$
\log \zeta(\theta)=\log \left[2 \varrho_{1}(\theta) \varrho_{1}(\pi-\theta)\right]+\sum_{k=-\infty}^{\infty} c_{k} \exp (i 2 v \theta k / \tau) .
$$

Using a Fourier expansion for theta functions which converges in the region (10.1) (see [31, Sect. 13.19])

$$
\begin{aligned}
\log \left[\frac{\vartheta_{1}(v(\pi-\theta) / \tau \mid-1 / 2 \tau)}{\vartheta_{1}(v \theta / \tau \mid-1 / 2 \tau)}\right]= & i \frac{v(\pi-2 \theta)}{\tau}+2 i \sum_{k=1}^{\infty} \frac{\sin [\pi(1-2 v) k / 2 \tau]}{k \sin (\pi k / 2 \tau)} \\
& \cdot \sin [v(\pi-2 \theta) k / \tau],
\end{aligned}
$$

and Eqs. (10.10) and (10.11), we obtain an analogous expansion for $\log \varphi(\theta)$ :

$$
\log \varphi(\theta)=2 i v \theta / \tau+\sum_{k=-\infty}^{\infty} b_{k} \exp (i 2 v \theta k / \tau) .
$$

Here $b_{k}$ are related to $c_{k}$ in the following manner:

$$
\begin{aligned}
& b_{0}=c_{0}-i \pi v(1-2 v) / \tau \\
& b_{k}=c_{k}+\exp (-i \pi v k / \tau) \frac{\sin [\pi(1-2 v) k / 2 \tau]}{k \sin (\pi k / 2 \tau)}, \quad k \neq 0 .
\end{aligned}
$$


The Fourier series (10.13) is supposed to converge in the interval $-\pi<\theta<\pi$ that will be justified by subsequent calculations.

Equations (10.9) and (4.9) after substitution (10.10) read:

$$
\begin{aligned}
\log \varphi(\pi-\theta)-\log \varphi(\theta)= & i 2 v(\pi-2 \theta) / \tau+4 i \sum_{k=1}^{\infty} \frac{\sin [\pi(1-2 v) k / 2 \tau]}{k \sin (\pi k / 2 \tau)} \\
& \cdot \sin [v(\pi-2 \theta) k / \tau], \\
\log \varphi(\theta)+\log \varphi(-\theta)= & 0 .
\end{aligned}
$$

Inserting the expansion (10.13) into Eqs. (10.15) and (10.16) and comparing Fourier coefficients on both sides of the system, we get

$$
b_{k}=\frac{\sin [\pi(1-2 v) k / 2 \tau]}{k \sin (\pi k / 2 \tau) \cos (\pi v k / \tau)} \quad \text { for } \quad k \neq 0, \quad b_{0}=0 .
$$

Now one can readily verify that the series in (10.13) is really convergent for $-\pi<\theta<\pi$.

Finally, inserting (10.17) into Eq. (10.14) to find coefficients $c_{k}$, we obtain that for $v<1 / 4,0<\theta<\pi$

$$
\begin{aligned}
\log \zeta(\theta)= & -\beta F_{A T}(\theta)=\log \left[2 \varrho_{1}(\theta) \varrho_{1}(\pi-\theta)\right]+i \pi v(1-2 v) / \tau \\
& +2 i \sum_{k=1}^{\infty} \frac{\sin [\pi(1-2 v) k / 2 \tau]}{k \sin (\pi k / 2 \tau)} \\
& \cdot \tan (\pi v k / \tau) \cos [v(\pi-2 \theta) k / \tau] .
\end{aligned}
$$

The following natural relationship exists for the free energy of the AshkinTeller model and the same quantity of the eight-vertex model:

$$
-\beta F_{A T}(\theta)=-2 \beta f_{8 V}(\theta),
$$

where the expression for $-\beta f_{8 V}(\theta)$ was obtained by Baxter [14]:

$$
\begin{aligned}
-\beta f_{8 V}(\theta)= & \log R_{0}^{0}(\theta)-4 i \sum_{k=1}^{\infty} \frac{\sin ^{2}[\pi(1-2 v) k / 2 \tau]}{k \sin (\pi k / \tau) \cos (\pi v k / \tau)} \\
& \cdot \sin (v \theta k / \tau) \sin [v(\pi-\theta) k / \tau] .
\end{aligned}
$$

The Ising model (Sect. 8). Quasi-periodicity of SW (8.14) causes the same quasiperiodicity of TM:

$$
\begin{gathered}
\mathbf{T}(\theta+2 \pi)=\left[-x(\pi+\theta) \cdot x^{-1}(\theta)\right]^{N} \mathbf{V} \mathbf{T}(\theta), \quad \mathbf{T}(\theta+4 \pi \tau)=\mathbf{T}(\theta), \\
\mathbf{V}_{\{\alpha, \beta\}}=\prod_{i=1}^{N} \delta_{\alpha_{i}+1, \beta_{i}} .
\end{gathered}
$$

Just the same reasoning as in the case of the Ashkin-Teller model applied to the case of the Ising model allows us to suggest the same hypothesis concerning periodicity of the eigenvalues with the period $4 \pi \tau$ instead of $\pi \tau / \nu$ in Eq. (10.7). Note that Eq. (8.16) is a particular form of Eq. (9.13) after the following substitution:

$$
2 \varrho_{1}(\theta) \varrho_{1}(\pi-\theta)=\vartheta_{2}(0 \mid \tau) / \vartheta_{2}(\pi / 4 \mid \tau) ; \quad v=1 / 8, \quad \tau \rightarrow \tau / 2 .
$$


Thus, Ising free energy may be extracted from Eq. (10.18) by the above substitution (10.22):

$$
\begin{aligned}
-\beta F_{I}(\theta)= & \log \left[\frac{\vartheta_{2}(0 \mid \tau)}{\vartheta_{2}(\pi / 4 \mid \tau)}\right]+3 \pi i / 16 \tau+2 i \sum_{k=1}^{\infty} \frac{\sin (3 \pi k / 4 \tau)}{k \sin (\pi k / \tau)} \\
& \cdot \tan (\pi k / 4 \tau) \cos [(\pi-2 \theta) k / 4 \tau] .
\end{aligned}
$$

Potts models $n>4$. Parameter $\mu$ in the solution (7.6) is real according to its definition (A.10). SW (7.1), (7.6) possess a pure imaginary period $\Omega=i \pi / \mu$. Equation (7.9) results from (10.9) after the Ashkin-Teller parameters are substituted as follows

$$
v=-i \mu \tau, \quad 2 \varrho_{1}(\theta) \varrho_{1}(\pi-\theta)=n^{1 / 2} \exp \left(-2 i \pi \tau \mu^{2}\right),
$$

and subsequently the limit $\tau \rightarrow 0$ is taken. The above substitution and subsequent pass to the limit $\tau=0$ do not violate the periodicity (10.8) with the above period $\Omega$. This allows us to conclude that the expression for the critical Potts $(n>4)$ free energy may be extracted from that of the Ashkin-Teller model (10.18) by the above substitution and proceeding to the limit $\tau \rightarrow 0(\operatorname{Im} \tau>0)$

$$
\begin{aligned}
-\beta F_{n}(\theta)= & \frac{1}{2} \log n+\mu \pi+2 \sum_{k=1}^{\infty} k^{-1} \exp (-\mu \pi k) \tanh (\mu \pi k) \\
& \cdot \cosh [\mu(\pi-2 \theta) k], \\
& n>4, \quad 0<\theta<\pi, \quad \mu>0, \quad 2 \cosh \mu \pi=n^{1 / 2} .
\end{aligned}
$$

Potts models $n<4$. Designate $\mu=i \gamma(\gamma \geqq 0)$. SW (7.6) in this case possess only a real period $\pi / \gamma$. However, we assume the aperiodicity in the second direction to be a limit of an infinitely large imaginary period of SW (7.6). This assumption allows us to obtain the solution of functional equations for $\log \zeta(\theta)$ from (10.18) substituting

$$
v=\gamma / 2, \quad 2 \varrho_{1}(\theta) \varrho_{1}(\pi-\theta)=n^{1 / 2},
$$

and subsequently passing to the $\operatorname{limit} \operatorname{Im} \tau \rightarrow+\infty$.

Substitution (10.26) alters the form of (10.18) in the following way:

$$
\begin{aligned}
\log \zeta(\theta)= & \frac{1}{2} \log n+\sum_{k=-\infty}^{\infty} \frac{\sinh \left[\frac{\pi(1-\gamma) k}{(-2 i \tau)}\right]}{\sinh \left[\frac{\pi k}{(-2 i \tau)}\right]} \tanh \left[\frac{\pi \gamma k}{(-2 i \tau)}\right] \\
& \cdot \cosh \left[\frac{\gamma(\pi-2 \theta) k}{(-2 i \tau)}\right] \frac{\frac{1}{(-2 i \tau)}}{\frac{k}{(-2 i \tau)}} .
\end{aligned}
$$

The last expression in (10.27) in the limit $\operatorname{Im} \tau \rightarrow \infty$ coincides with the usual definition of the Riemann integral:

$$
\begin{aligned}
-\beta F_{n}(\theta)= & \frac{1}{2} \log n+\int_{-\infty}^{\infty} \frac{d x}{x} \frac{\sinh [\pi(1-\gamma) x]}{\sinh (\pi x)} \tanh (\pi \gamma x) \cosh [\gamma(\pi-2 \theta) x], \\
& n \leqq 4, \quad 0<\theta<\pi, \quad 0 \leqq \gamma \leqq 1 / 2, \quad 2 \cos \gamma \pi=n^{1 / 2} .
\end{aligned}
$$


Potts model $n=4$ is a special case corresponding to the limit $\gamma \rightarrow 0$ in Eq. (7.6). Substituting the variable in Eq. (10.28) $x=z / \gamma$ and proceeding to the limit $\gamma \rightarrow 0$, one gets the expression for the free energy in the present case:

$$
\begin{aligned}
-\beta F_{4}(\theta) & =\log 2+2 \int_{0}^{\infty} \frac{d z}{z} \exp (-\pi z) \tanh (\pi z) \cosh [(\pi-2 \theta) z] \\
& =\log 2+\log \left[\frac{\theta(\pi-\theta)}{(2 \pi)^{2}}\right]+2 \log \left[\frac{\Gamma(\theta / 2 \pi) \Gamma(1 / 2-\theta / 2 \pi)}{\Gamma(1 / 2+\theta / 2 \pi) \Gamma(1-\theta / 2 \pi)}\right](10.29)
\end{aligned}
$$

Here an integral representation for $\log \Gamma(x)$ [31, Sect. 1.9] is used. The formulas (10.27)-(10.29) coincide with the corresponding expressions by Baxter [39] who exploited the equivalence of the partition functions for the Potts models and the staggered six-vertex model $[37,38]$.

\section{Appendix A}

This appendix is devoted to a solution of functional equations (7.2)-(7.4). At first consider Eq. (7.3) written in the following form:

$$
v\left(\theta_{3}\right) v\left(\pi-\theta_{3}\right)=\lambda^{-1}\left(\theta_{1}, \theta_{2}, \theta_{3}\right) v\left(\theta_{1}\right) v\left(\theta_{2}\right) v\left(\theta_{3}\right) .
$$

The left-hand side of Eq. (A.1) does not depend on $\theta_{1}$ while its right-hand side is a symmetric function of $\theta_{1}, \theta_{2}, \theta_{3}$ provided $\theta_{1}+\theta_{2}+\theta_{3}=\pi$, so both sides cannot depend on $\theta_{2}, \theta_{3}$ either, being equal constants:

$$
\begin{gathered}
v(\theta) v(\pi-\theta)=a, \\
\lambda\left(\theta_{1}, \theta_{2}, \theta_{3}\right)=a^{-1} v\left(\theta_{1}\right) v\left(\theta_{2}\right) v\left(\theta_{3}\right) .
\end{gathered}
$$

Having expressed $v(\pi-\theta)$ via $v(\theta)$ with the help of (A.2), inserting this expression into Eq. (7.4) and using Eq. (A.3) simultaneously the following relationship may be obtained:

$$
\lambda\left(\theta_{1}, \theta_{2}, \theta_{3}\right)=a+v\left(\theta_{1}\right)+v\left(\theta_{2}\right)+v\left(\theta_{3}\right)=a^{-1} v\left(\theta_{1}\right) v\left(\theta_{2}\right) v\left(\theta_{3}\right) .
$$

A comparison of Eq. (A.4) with Eq. (7.2) yields the value of $a=n$. Now Eqs. (7.2) and (A.3) with the condition $\theta_{3}=\pi-\theta_{1}-\theta_{2}$ allow us to derive an addition theorem for the function $v(\theta)$ :

$$
v\left(\theta_{1}+\theta_{2}\right)=\frac{v\left(\theta_{1}\right) v\left(\theta_{2}\right)-n}{n+v\left(\theta_{1}\right)+v\left(\theta_{2}\right)} .
$$

The following linear-fractional substitution

$$
v(\theta)=\frac{1}{2} n^{1 / 2} \frac{\left[(n-4)^{1 / 2}-n^{1 / 2}\right] u(\theta)+\left[(n-4)^{1 / 2}+n^{1 / 2}\right]}{u(\theta)-1}
$$

converts the addition theorem (A.5) into a trivial one:

$$
u\left(\theta_{1}+\theta_{2}\right)=u\left(\theta_{1}\right) u\left(\theta_{2}\right) .
$$

Note that $\operatorname{SBC}(7.5)$ for $v(\theta)$ in terms of $u(\theta)$ read:

$$
u(0)=1, \quad u(\pi)=\frac{n^{1 / 2}+(n-4)^{1 / 2}}{n^{1 / 2}-(n-4)^{1 / 2}} .
$$


The unique solution of (A.8) is obviously

$$
u(\theta)=\exp (2 \mu \theta),
$$

where the parameter $\mu$ is fixed by the second $\mathrm{SBC}$ (A.8):

$$
n^{1 / 2}=2 \cosh \mu \pi \text {. }
$$

Inserting Eqs. (A.9) and (A.10) into (A.6) one gets a final answer for $v(\theta)$ :

$$
v(\theta)=2 \cosh \mu \pi \frac{\sinh \mu(\pi-\theta)}{\sinh \mu \theta} .
$$

\section{Appendix B}

STR (8.1) and (8.2) are solved in this appendix. Eliminating $\lambda\left(\theta_{1}, \theta_{2}, \theta_{3}\right)$ from Eqs. (8.1), (8.2) one comes to a single equation

$$
\frac{1+x\left(\theta_{1}\right) x\left(\theta_{2}\right) x\left(\theta_{3}\right)}{x\left(\theta_{1}\right)+x\left(\theta_{2}\right) x\left(\theta_{3}\right)}=x\left(\pi-\theta_{2}\right) x\left(\pi-\theta_{3}\right),
$$

provided $\theta_{1}+\theta_{2}+\theta_{3}=\pi$ and any permutation of $\theta_{1}, \theta_{2}, \theta_{3}$ is admissible. Since $x(\theta)=K_{0}(\theta)$ and the normalization $K_{1}(\theta)=1$ is adopted, SBC (2.20) for $x(\theta)$ read: $x(\pi)=1$ and $\lim _{\theta \rightarrow 0} x(\theta)=\infty$. We find it convenient to introduce the function

$$
y(\theta)=x(\pi-\theta) \text {. }
$$

Equation (B.1) may be rewritten as a system of two functional equations for two functions $x(\theta)$ and $y(\theta)$ :

$$
\begin{aligned}
& 1+x\left(\theta_{1}\right) x\left(\theta_{2}\right) y\left(\theta_{1}+\theta_{2}\right)=y\left(\theta_{1}\right) y\left(\theta_{2}\right)\left[y\left(\theta_{1}+\theta_{2}\right)+x\left(\theta_{1}\right) x\left(\theta_{2}\right)\right], \\
& 1+x\left(\theta_{1}\right) x\left(\theta_{2}\right) y\left(\theta_{1}+\theta_{2}\right)=x\left(\theta_{1}+\theta_{2}\right) y\left(\theta_{2}\right)\left[x\left(\theta_{1}\right)+y\left(\theta_{1}+\theta_{2}\right) x\left(\theta_{2}\right)\right] .
\end{aligned}
$$

Having expressed $x\left(\theta_{2}\right)$ from (B.3) and having passed to the limit $\theta_{2} \rightarrow 0$, one readily sees that for any given $\theta_{1}$

$$
\lim _{\theta \rightarrow 0}[\theta x(\theta)]=x^{-1}\left(\theta_{1}\right)\left[y^{\prime}\left(\theta_{1}\right)-y^{\prime}(0) y\left(\theta_{1}\right)\right]^{-1}\left[y^{2}\left(\theta_{1}\right)-1\right] .
$$

The possibility $y^{2}(\theta)=1$ contradicts the SBC $\lim _{\theta \rightarrow \pi} y(\theta)=\infty$, and another possibility $y^{\prime}(\theta)=y^{\prime}(0) y(\theta)$ due to SBC $y(0)=1$ means $y(\theta)=\exp \left[y^{\prime}(0) \theta\right]$ and contradicts the same SBC. Thus, Eq. (B.5) leaves the only variant of singularity for $x(\theta)$ at $\theta \rightarrow 0$ that is a simple pole. Denote the residue in that pole by $\alpha$

$$
\left.x(\theta)\right|_{\theta \rightarrow 0}=\alpha / \theta+O(1),
$$

and the derivative $y^{\prime}(0)$ by $\beta$. Then Eq. (B.5) combined with (B.6) gives a differential equation

$$
y^{\prime}(\theta)=\beta y(\theta)+\frac{y^{2}(\theta)-1}{\alpha x(\theta)} .
$$

Another differential equation may be obtained in the limit $\theta_{2} \rightarrow 0$ from (B.4):

$$
x^{\prime}(\theta)=-\beta x(\theta)-\frac{x^{2}(\theta)-1}{\alpha y(\theta)} .
$$


The latter is equivalent to Eq. (B.7) on account of the condition (B.2). Another limit $\theta_{1} \rightarrow 0$ in the same Eq. (B.4) leads to the third differential equation

$$
x(\theta) y^{\prime}(\theta)-x^{\prime}(\theta) y(\theta)=\alpha^{-1}\left[x^{2}(\theta) y^{2}(\theta)-1\right] .
$$

Substituting the expressions for the derivatives $x^{\prime}(\theta)$ and $y^{\prime}(\theta)$ from Eqs. (B.7) and (B.8), respectively, into Eq. (B.9) one may get an algebraic relationship between $x(\theta)$ and $y(\theta)$

$$
\left[x^{2}(\theta)-1\right]\left[y^{2}(\theta)-1\right]=2 \alpha \beta x(\theta) y(\theta) .
$$

Squaring both sides of Eq. (B.8) and using Eq. (B.10) one can eliminate $y(\theta)$ :

$$
x^{\prime}(\theta)=-\alpha^{-1}\left[x^{4}(\theta)+\left(\alpha^{2} \beta^{2}-2\right) x^{2}(\theta)+1\right]^{1 / 2} .
$$

The sign minus is chosen here in agreement with Eq. (B.6).

Equation (B.1) may be solved in terms of Jacobi elliptic functions [31, Sects. 13.9, 13.17]. Two parameters $k_{1}$ and $\lambda$ are better suited for this solution than $\alpha$ and $\beta$ :

$$
k_{1}+1 / k_{1}=\alpha^{2} \beta^{2}-2, \quad \lambda^{-1}=\alpha k_{1}^{1 / 2} .
$$

The solution of Eq. (B.11) may be expressed via elliptic functions having the modulus $k=\left(1-k_{1}^{2}\right)^{1 / 2}$ complementary to $k_{1}$ [31, Sect. 13.17]:

$$
x(\theta)=k_{1}^{-1 / 2} \operatorname{cs}(\lambda \theta \mid k) .
$$

SBC $x(\pi)=1$ fixes $\lambda=\frac{K}{2 \pi}, K$ is a complete elliptic integral of modulus $k$ :

$$
x(\theta)=k_{1}^{-1 / 2} \operatorname{cs}(K \theta / 2 \pi \mid k)=\vartheta_{2}(\theta / 4 \mid \tau) / \vartheta_{1}(\theta / 4 \mid \tau),
$$

where $\vartheta_{1}(z \mid \tau)$ and $\vartheta_{2}(z \mid \tau)$ are conventional Jacobi theta functions [31, Sect. 13.19$]^{4}$.

The solution of differential equations (B.13) satisfies the functional relation (B.1) at the same time. The simplest proof of this fact is based on Liouville's theorem. Both sides of Eq. (B.1) due to Eq. (B.13) are double-periodic functions of $\theta_{2}$ provided $\theta_{3}$ is fixed $\left(\theta_{1}=\pi-\theta_{2}-\theta_{3}\right)$. A comparison of zeros and poles of these functions shows their coincidence. Thus, both functions may differ only by a multiplicative constant. Putting $\theta_{2}=0$ one maintaines its value equal to unity. The proof is thus completed. We do not wish to present the details here because of the lack of space.

\section{Appendix C}

In this appendix some formulas concerning the eight-vertex model are collected. On one hand, the aim is to illustrate the general theorems of Sect. 5 and on the other hand to apply these calculations to derive some results needed in Sect. 9.

The eight-vertex model in the notation of Sect. 5 is the $Z_{2} \times Z_{2}$-symmetric model described by the matrix $R_{\mu}^{\sigma}(\theta)$ where $\sigma$ and $\mu$ take the values 0,1 . There are 
only two spin configurations leading to different functional equations (2.14) for SW (5.1):

$$
\begin{gathered}
\sigma_{1}=\sigma_{2}=\sigma_{3}, \quad \mu_{1} \neq \mu_{2}=\mu_{3}, \\
R_{0}^{0}\left(\theta_{1}\right) R_{1}^{0}\left(\theta_{2}\right) R_{1}^{0}\left(\theta_{3}\right)+R_{0}^{1}\left(\theta_{1}\right) R_{1}^{1}\left(\theta_{2}\right) R_{1}^{1}\left(\theta_{3}\right) \\
=R_{0}^{0}\left(\theta_{1}\right) R_{0}^{1}\left(\theta_{2}\right) R_{0}^{1}\left(\theta_{3}\right)+R_{0}^{1}\left(\theta_{1}\right) R_{0}^{0}\left(\theta_{2}\right) R_{0}^{0}\left(\theta_{3}\right),
\end{gathered}
$$

and

$$
\begin{gathered}
\sigma_{1}=\sigma_{2} \neq \sigma_{3}, \quad \mu_{1} \neq \mu_{2}=\mu_{3}, \\
R_{0}^{0}\left(\theta_{1}\right) R_{1}^{0}\left(\theta_{2}\right) R_{1}^{1}\left(\theta_{3}\right)+R_{0}^{1}\left(\theta_{1}\right) R_{1}^{1}\left(\theta_{2}\right) R_{1}^{0}\left(\theta_{3}\right) \\
=R_{1}^{1}\left(\theta_{1}\right) R_{1}^{0}\left(\theta_{2}\right) R_{0}^{0}\left(\theta_{3}\right)+R_{1}^{0}\left(\theta_{1}\right) R_{1}^{1}\left(\theta_{2}\right) R_{0}^{1}\left(\theta_{3}\right) .
\end{gathered}
$$

Any other equation of the system (2.14) that does not degenerate into an identity reduces to either Eqs. (C.1) or (C.2) by some transposition of angles $\theta_{1}, \theta_{2}, \theta_{3}$.

The crossing condition (2.13)

$$
R_{0}^{0}(\theta)=R_{0}^{0}(\pi-\theta), \quad R_{1}^{1}(\theta)=R_{1}^{1}(\pi-\theta), \quad R_{0}^{1}(\theta)=R_{1}^{0}(\pi-\theta),
$$

together with $\operatorname{SBC}(2.15)$

$$
R_{0}^{0}(0)=R_{1}^{0}(0)=1, \quad R_{0}^{1}(0)=R_{1}^{1}(0)=0
$$

determines a unique solution of Eqs. (C.1), (C.2) up to an arbitrary normalization factor $\varrho(\theta)$ obeying, however, both conditions (C.3), (C.4). That means $\varrho(\theta)=\varrho(\pi-\theta)$ and $\varrho(0)=1$. The above freedom takes its origin in the uniformity of the system (2.14) both with SBC (2.15) and the crossing condition (2.13).

The solution becomes unambiguous if normalization $R_{0}^{0}(\theta)=1$ is assumed. It was obtained by Baxter $[13,14]$ who manipulated purely algebraically and by Zamolodchikov [15] who, assuming in turn $\theta_{1}, \theta_{2}, \theta_{3}$ infinitesimal and linearizing Eqs. (C.1), (C.2), came to a system of differential equations instead of functional ones. The reader who is interested in details may find them in the above references. Here we quote the result:

$$
R_{0}^{0}(\theta)=1,
$$

$$
\begin{gathered}
R_{0}^{1}(\theta)=R_{1}^{0}(\pi-\theta)=\frac{\operatorname{sn}\left(\lambda_{1} \theta \mid k_{1}\right)}{\operatorname{sn}\left(\lambda_{1} \pi \mid k_{1}\right)}=\frac{\vartheta_{4}\left(v_{1} \pi \mid \tau_{1}\right) \vartheta_{1}\left(v_{1} \theta \mid \tau_{1}\right)}{\vartheta_{1}\left(v_{1} \pi \mid \tau_{1}\right) \vartheta_{4}\left(v_{1} \theta \mid \tau_{1}\right)}, \\
R_{1}^{1}(\theta)=-k_{1} \operatorname{sn}\left(\lambda_{1} \theta \mid k_{1}\right) \operatorname{sn}\left(\lambda_{1}(\pi-\theta) \mid k_{1}\right)=-\frac{\vartheta_{1}\left(v_{1} \theta \mid \tau_{1}\right) \vartheta_{1}\left(v_{1}(\pi-\theta) \mid \tau_{1}\right)}{\vartheta_{4}\left(v_{1} \theta \mid \tau_{1}\right) \vartheta_{4}\left(v_{1}(\pi-\theta) \mid \tau_{1}\right)},
\end{gathered}
$$

where $\lambda_{1}=2 K\left(k_{1}\right) v_{1} / \pi$ and $\tau_{1}=i K^{\prime}\left(k_{1}\right) / K\left(k_{1}\right)$. Here $K(k)$ and $K^{\prime}(k)$ denote elliptic integrals of modulus $k$ (see [31, Sect. 13.20]).

In subsequent calculations we return to an arbitrary normalization instead of $R_{0}^{0}(\theta)=1$ and make a modular Jacobi transformation [31, Sect. 13.22]

$$
\tau=-1 / \tau_{1}, \quad v=-v_{1} / \tau_{1} .
$$

The resulting form of SW we use is the following:

$$
\begin{aligned}
& R_{0}^{0}(\theta)=\varrho(\theta \mid \tau) \vartheta_{2}(0 \mid \tau) \vartheta_{1}(v \pi \mid \tau) \vartheta_{2}(v \theta \mid \tau) \vartheta_{2}(v(\pi-\theta) \mid \tau), \\
& R_{1}^{0}(\theta)=\varrho(\theta \mid \tau) \vartheta_{2}(0 \mid \tau) \vartheta_{2}(v \pi \mid \tau) \vartheta_{2}(v \theta \mid \tau) \vartheta_{1}(v(\pi-\theta) \mid \tau), \\
& R_{0}^{1}(\theta)=\varrho(\theta \mid \tau) \vartheta_{2}(0 \mid \tau) \vartheta_{2}(v \pi \mid \tau) \vartheta_{1}(v \theta \mid \tau) \vartheta_{2}(v(\pi-\theta) \mid \tau), \\
& R_{1}^{1}(\theta)=\varrho(\theta \mid \tau) \vartheta_{2}(0 \mid \tau) \vartheta_{1}(v \pi \mid \tau) \vartheta_{1}(v \theta \mid \tau) \vartheta_{1}(v(\pi-\theta) \mid \tau) .
\end{aligned}
$$


DT (5.5) converts the solution (C.7) into another one according to Theorem 1 of Sect. 5. Dual weights $R_{\beta}^{\sim \alpha}(\theta)$ should be expressed via $R_{\beta}^{\alpha}(\theta)($ C.7) according to Eq. (5.5) with $\omega=-1$. First, we prefer to deduce some intermediate formulas useful both for DT and for the Ashkin-Teller model (Sect. 9). These formulas were previously mentioned as partial DT (5.10) where $\omega=-1, n=2$. Using addition formulas for Jacobi theta functions ${ }^{5}$

$$
\begin{aligned}
& \vartheta_{2}(x+y \mid \tau) \vartheta_{2}(x-y \mid \tau)+\vartheta_{1}(x+y \mid \tau) \vartheta_{1}(x-y \mid \tau)=2 \vartheta_{3}(2 x \mid 2 \tau) \vartheta_{2}(2 y \mid 2 \tau), \\
& \vartheta_{1}(x+y \mid \tau) \vartheta_{2}(x-y \mid \tau)+\vartheta_{2}(x+y \mid \tau) \vartheta_{1}(x-y \mid \tau)=2 \vartheta_{1}(2 x \mid 2 \tau) \vartheta_{4}(2 y \mid 2 \tau),
\end{aligned}
$$

for Eq. (C.7) substituted into Eq. (5.10) we obtain:

$$
\begin{aligned}
& K_{00}(\theta)=2^{3 / 2} \varrho(\theta \mid \tau) \vartheta_{2}(v \theta \mid 2 \tau) \vartheta_{3}(v \theta \mid 2 \tau) \vartheta_{4}(v \theta \mid 2 \tau) \vartheta_{1}(v(2 \pi-\theta) \mid 2 \tau), \\
& K_{01}(\theta)=2^{3 / 2} \varrho(\theta \mid \tau) \vartheta_{1}(v \theta \mid 2 \tau) \vartheta_{2}(v \theta \mid 2 \tau) \vartheta_{4}(v \theta \mid 2 \tau) \vartheta_{3}(v(2 \pi-\theta) \mid 2 \tau), \\
& K_{10}(\theta)=2^{3 / 2} \varrho(\theta \mid \tau) \vartheta_{1}(v \theta \mid 2 \tau) \vartheta_{2}(v \theta \mid 2 \tau) \vartheta_{3}(v \theta \mid 2 \tau) \vartheta_{4}(v(2 \pi-\theta) \mid 2 \tau) \\
& K_{11}(\theta)=2^{3 / 2} \varrho(\theta \mid \tau) \vartheta_{1}(v \theta \mid 2 \tau) \vartheta_{3}(v \theta \mid 2 \tau) \vartheta_{4}(v \theta \mid 2 \tau) \vartheta_{2}(v(2 \pi-\theta) \mid 2 \tau)
\end{aligned}
$$

Now perform a modular transformation $2 \tau \rightarrow 2 \tau+1$ [31, Sect. 13.22] that leads to an exchange of $K_{01}(\theta)$ and $K_{10}(\theta)$ neglecting the change of $\varrho(\theta \mid \tau)$. This allows us to use Eq. (C.8) once more in the form

$$
\begin{aligned}
& \vartheta_{2}(x+y \mid 2 \tau) \vartheta_{3}(x-y \mid 2 \tau)+\vartheta_{3}(x+y \mid 2 \tau) \vartheta_{2}(x-y \mid 2 \tau)=\vartheta_{2}(x \mid \tau) \vartheta_{2}(y \mid \tau), \\
& \vartheta_{2}(x+y \mid 2 \tau) \vartheta_{3}(x-y \mid 2 \tau)-\vartheta_{3}(x+y \mid 2 \tau) \vartheta_{2}(x-y \mid 2 \tau)=-\vartheta_{1}(x \mid \tau) \vartheta_{1}(y \mid \tau), \\
& \vartheta_{1}(x+y \mid 2 \tau) \vartheta_{4}(x-y \mid 2 \tau)+\vartheta_{4}(x+y \mid 2 \tau) \vartheta_{1}(x-y \mid 2 \tau)=\vartheta_{1}(x \mid \tau) \vartheta_{2}(y \mid \tau), \\
& \vartheta_{1}(x+y \mid 2 \tau) \vartheta_{4}(x-y \mid 2 \tau)-\vartheta_{4}(x+y \mid 2 \tau) \vartheta_{1}(x-y \mid 2 \tau)=\vartheta_{2}(x \mid \tau) \vartheta_{1}(y \mid \tau) .
\end{aligned}
$$

The final formulas for dual SW read:

$$
\begin{aligned}
& R_{0}^{\sim 0}(\theta)=-i \varrho(\theta \mid \tau) \vartheta_{2}(0 \mid \tau+1 / 2) \vartheta_{1}(v \pi \mid \tau+1 / 2) \vartheta_{2}(v \theta \mid \tau+1 / 2) \vartheta_{2}(v(\pi-\theta) \mid \tau+1 / 2), \\
& R_{1}^{\sim 0}(\theta)=-i \varrho(\theta \mid \tau) \vartheta_{2}(0 \mid \tau+1 / 2) \vartheta_{2}(v \pi \mid \tau+1 / 2) \vartheta_{2}(v \theta \mid \tau+1 / 2) \vartheta_{1}(v(\pi-\theta) \mid \tau+1 / 2), \\
& R_{0}^{\sim 1}(\theta)=-i \varrho(\theta \mid \tau) \vartheta_{2}(0 \mid \tau+1 / 2) \vartheta_{2}(v \pi \mid \tau+1 / 2) \vartheta_{1}(v \theta \mid \tau+1 / 2) \vartheta_{2}(v(\pi-\theta) \mid \tau+1 / 2), \\
& R_{1}^{\sim 1}(\theta)=-i \varrho(\theta \mid \tau) \vartheta_{2}(0 \mid \tau+1 / 2) \vartheta_{1}(v \pi \mid \tau+1 / 2) \vartheta_{1}(v \theta \mid \tau+1 / 2) \vartheta_{1}(v(\pi-\theta) \mid \tau+1 / 2)
\end{aligned}
$$

These expressions may be summarized in the following way:

$$
\varrho^{-1}(\theta \mid \tau) R_{\beta}^{\sim \alpha}(\theta \mid \tau)=-i \varrho^{-1}(\theta \mid \tau+1 / 2) R_{\beta}^{\alpha}(\theta \mid \tau+1 / 2) .
$$

An important invariant (i.e. a quantity independent of $\theta$ ) may be constructed by virtue of Eqs. (C.7) and (C.11):

$$
\frac{R_{0}^{\sim 0}(\theta \mid \tau) R_{0}^{\sim 1}(\theta \mid \tau) R_{1}^{\sim 0}(\theta \mid \tau) R_{1}^{\sim 1}(\theta \mid \tau)}{R_{0}^{0}(\theta \mid \tau) R_{0}^{1}(\theta \mid \tau) R_{1}^{0}(\theta \mid \tau) R_{1}^{1}(\theta \mid \tau)}=\frac{\vartheta_{4}^{6}(0 \mid 2 \tau+1) \vartheta_{2}^{4}(0 \mid \tau+1 / 2)}{i \vartheta_{4}^{6}(0 \mid 2 \tau) \vartheta_{2}^{4}(0 \mid \tau)}=\frac{\vartheta_{3}^{4}(0 \mid 2 \tau)}{\vartheta_{4}^{4}(0 \mid 2 \tau)}
$$

Here we used Eq. (C.10) several times: the third equation to obtain the first equality (C.13) and then the first formula for $x=y=0$. Modular transformation $2 \tau+1 \rightarrow 2 \tau[31$, Sect. 13.22] completes the calculation.

5 These formulas may be established by direct algebraic manipulations with Fourier series for Jacobi theta functions [31, Sect. 13.19] 
By means of the invariant (C.13) parameter $\tau$ may be expressed via physical parameters of the model. The latter determine SW in the following way:

$$
R_{\gamma}^{\alpha}(\theta)=A(\theta) \exp \left[\beta_{1}(\theta)(-1)^{\alpha}+\beta_{2}(\theta)(-1)^{\gamma}+\beta_{3}(\theta)(-1)^{\alpha+\gamma}\right] .
$$

Inserting Eq. (C.14) into Eq. (C.13) and using Eq. (5.5) we obtain:

$$
\begin{aligned}
& \vartheta_{3}^{4}(0 \mid 2 \tau) / \vartheta_{4}^{4}(0 \mid 2 \tau)=\sinh ^{2} 2 \beta_{1} \cdot \sinh ^{2} 2 \beta_{2}+\sinh ^{2} 2 \beta_{2} \cdot \sinh ^{2} 2 \beta_{3}+\sinh ^{2} 2 \beta_{3} \cdot \sinh ^{2} 2 \beta_{1} \\
& \quad+2 \sinh 2 \beta_{1} \cdot \sinh 2 \beta_{2} \cdot \sinh 2 \beta_{3}\left(\cosh 2 \beta_{1} \cdot \cosh 2 \beta_{2} \cdot \cosh 2 \beta_{3}\right. \\
& \left.\quad+\sinh 2 \beta_{1} \cdot \sinh 2 \beta_{2} \cdot \sinh 2 \beta_{3}\right) .
\end{aligned}
$$

Equation (C.15) plays the same role in the case of the eight-vertex model as Eq. (8.9) for the Ising model. A critical point is achieved when the right-hand side of (C.15) turns to unity [13,29]. In the vicinity of the critical point the relationship between $\tau$ and temperature becomes transparent. Just as in the case of Eq. (8.13), an expansion near $T_{c}$ gives

$$
\tau=\frac{1}{2 \pi i} \log \left(1-\frac{T}{T_{c}}\right)+O(1)
$$

in agreement with the general formula (C.12).

The above relationship (C.15) for the eight-vertex model is totally equivalent to Eq. (8.9) for the Ising model when the interaction of the Ising sublattices constituting the eight-vertex model vanishes. This is the $\beta_{3}=0$ case. In other words, that means $R_{0}^{0} R_{1}^{1}=R_{1}^{0} R_{0}^{1}$. This requirement imposed on Eq. (C.7) means

$$
\vartheta_{1}^{2}(v \pi \mid \tau)=\vartheta_{2}^{2}(v \pi \mid \tau) \text {. }
$$

The latter is fulfilled when $v=1 / 4$.

Acknowledgements. This work was initiated after several stimulating discussions with Prof. A. M. Polyakov. We appreciate the discussions of the Ashkin-Teller and $Z_{4}$ models with Prof. V. L. Pokrovsky. We are indebted to I. R. Arkhipova for her help in the preparation of the English text.

\section{References}

1. Onsager, L. : Phys. Rev. 65, 117 (1944)

2. Onsager, L. : Critical phenomena in alloys, magnets, and superconductors. Mills, Ascher, and Jaffe (eds.). New York: McGraw-Hill 1971

3. Houtappel, R.M.F.: Physica 16, 425 (1950)

4. Husimi, K., Syozi, I.: Progr. Theor. Phys. 5, 177, 341 (1950)

5. Wannier, G.H.: Phys. Rev. 79, 357 (1950)

6. Stephenson, J.: J. Math. Phys. 5, 1009 (1964)

7. Baxter, R.J., Enting, I.G.: J. Phys. A 11, 2463 (1978)

8. Hilhorst, H.J., Schick, M., van Leeunwen, J.M.J.: Phys. Rev. B 19, 2749 (1979)

9. Knops, J.F., Hilhorst, H.J.: Phys. Rev. B 19, 3689 (1979)

10. Stephen, M.J., Mittag, L.: Phys. Lett. 41 A, 357 (1972)

11. Potts, R.B.: Proc. Camb. Philos. Soc. 48, 106 (1952)

12. Yang, C.N. : Phys. Rev. 168, 1920 (1968)

13. Baxter, R.J.: Ann. Phys. 70, 193 (1972)

14. Baxter, R.J.: Philos. Trans. R. Soc. 289, 315 (1978)

15. Zamolodchikov, A.B.: Commun. Math. Phys. 69, 165 (1979)

16. Sklyanin, Ye.K., Tahtadjan, L.A., Faddeev, L.D.: TMF 40, 194 (1979)

17. Faddeev, L.D. : Preprint LOMI P-2-79, Leningrad 1979

18. Kulish, P.P.: Preprint LOMI P-3-79, Leningrad 1979 
19. Stroganov, Yu. : Phys. Lett. 74 A, 116 (1979)

20. Bashilov, Yu.A., Pokrovsky, S.V.: Commun. Math. Phys. 76, 129 (1980)

21. Zamolodchikov, A.B., Zamolodchikov, Al.B.: Ann. Phys. 120, 253 (1979); Phys. Lett. 72 B, 481 (1978); Nucl. Phys. B 133, 525 (1978)

22. Fateev, V.A.: Yad. Fiz. 33, 1419 (1981)

23. Belavin, A.A.: Pisma v ZhETF, 32, 182 (1980)

24. Stephen, M.J., Mittag, L.: J. Math. Phys. 13, 1944 (1972)

25. Gantmakher, F.R.: Theory of matrices. Moscow: Nauka 1967

26. Kramers, H.A., Wannier, G.H.: Phys. Rev. 60, 252 (1941)

27. Wegner, F.: J. Math. Phys. 12, 2259 (1971)

28. Dotzenko, V.S.: ZhETF 75, 1083 (1978)

29. Fan, C., Wu, F.Y.: Phys. Rev. B 2, 723 (1970)

30. Kadanoff, L.P., Wegner, F.: Phys. Rev. B4, 3989 (1971)

31. Bateman, H., Erdelyi, A.: Higher transcendental functions. New York, Toronto, London: McGraw-Hill Book Company, Inc. 1955

32. Rumer, Yu.B.: UFN 53, 247 (1954)

33. Mittag, L., Stephen, M.J.: J. Math. Phys. 12, 441 (1971)

34. Pokrovsky, S.V.: Landau Institute preprint, Chernogolovka, 1978

35. Lüscher, M.: Nucl. Phys. B 117, 475 (1976)

36. Baxter, R.J., Temperley, H.N.V., Ashley, S.E.: Proc. R. Soc. (Lond.) A 358, 539 (1978)

37. Temperley, H.N.V., Lieb, E.H.: Proc. R. Soc. (Lond.) A 322, 251 (1971)

38. Baxter, R.J., Kelland, S.B., Wu, F.Y.: J. Phys. A 9, 397 (1976)

39. Baxter, R.J.: J. Phys. C 6, L 445 (1973)

40. Ashkin, J., Teller, E. : Phys. Rev. 64, 178 (1943)

Communicated by Ya. G. Sinai

Received September 25, 1981 